Accepted 10 November, 1999; To appear in ApJ, Vol. 532

\title{
Radiation hydrodynamics of SN 1987A: I. Global analysis of the light curve for the first 4 months
}

\author{
Sergei Blinnikov ${ }^{1}$ \\ Institute for Theoretical and Experimental Physics, 117259, Moscow, Russia \\ Peter Lundqvist ${ }^{2}$ \\ Stockholm Observatory, SE-133 36 Saltsjöbaden, Sweden \\ Oleg Bartunov ${ }^{1}$ \\ Sternberg Astronomical Institute, 119899, Moscow, Russia \\ Ken'ichi Nomoto ${ }^{3}$ and Koichi Iwamoto ${ }^{3}$ \\ ${ }^{1}$ Department of Astronomy and the Research Center for the Early Universe, University of Tokyo, \\ Tokyo 113-0033, Japan
}

\begin{abstract}
The optical/UV light curves of SN 1987A are analyzed with the multi-energy group radiation hydrodynamics code STELLA. The calculated monochromatic and bolometric light curves are compared with observations shortly after shock breakout, during the early plateau, through the broad second maximum, and during the earliest phase of the radioactive tail. We have concentrated on a progenitor model calculated by Nomoto \& Hashimoto and Saio, Nomoto, \& Kato, which assumes that $14 M_{\odot}$ of the stellar mass is ejected. Using this model, we have updated constraints on the explosion energy and the extent of mixing in the ejecta. In particular, we determine the most likely range of $E / M$ (explosion energy over ejecta mass) and $R_{0}$ (radius of the progenitor). In general, our best models have energies in the range $E=(1.1 \pm 0.3) \times 10^{51} \mathrm{ergs}$, and the agreement is better than in earlier, flux-limited diffusion calculations for the same explosion energy. Our modeled $B$ and $V$ fluxes compare well with observations, while the flux in $U$ undershoots after $\sim 10$ days by a factor of a few, presumably due to NLTE and line transfer effects. We also compare our results with IUE observations, and a very good quantitative agreement is found for the first days, and for one IUE band $(2500-3000 \AA)$ as long as for 3 months. We point out that the $V$ flux estimated by McNaught \& Zoltowski should probably be revised to a lower value.
\end{abstract}

\footnotetext{
${ }^{1}$ E-mail: blinn@sai.msu.su, oleg@sai.msu.su.

${ }^{2}$ E-mail: peter@astro.su.se.

${ }^{3}$ E-mail: nomoto@astron.s.u-tokyo.ac.jp, iwamoto@astron.s.u-tokyo.ac.jp.
} 
Subject headings: supernovae: individual (SN 1987A) — hydrodynamics — radiative transfer — shock waves — stars: interiors — scattering — supernovae: general

\section{Introduction}

SN 1987A has provided us with an excellent opportunity to test the theory of massive star evolution, nucleosynthesis, and supernova explosion. The broad band photometric observations, ranging from ultraviolet to far-infrared, and the resultant bolometric light curve enable us to probe the physical processes occurring in the interior of SN 1987A.

The light curve is so sensitive to the hydrodynamics that it is a useful tool to infer the progenitor's radius, the distribution of elements, mass of the ejecta (in particular, the mass of the hydrogen-rich envelope, $M_{\text {env }}$ ), and the explosion energy, $E$ (e.g., Nomoto et al. 1994 for a review). Although earlier theoretical models with flux-limited diffusion approximation are generally in good agreement with observations (see, e.g., Arnett et al. 1989a, and Hillebrandt \& Höflich 1989 for a review), there are still some uncertainties. One of the most important complications is that the supernova atmosphere is scattering dominated so that the color temperature is much higher than the effective temperature; the spectrum is a superposition of spectra emerging from layers with different depths and temperatures (Imshennik \& Utrobin 1977; Shigeyama et al. 1987; Pizzochero 1990; Höflich \& Wheeler 1999 and references therein). This effect is crucial to include in order to constrain model parameters.

Previous works on modeling this effect have used supernova atmospheric codes which rely either on the temperature structure derived from equilibrium diffusion models (Höflich 1991), or on the time-dependent luminosity from one-group radiation-hydro models (Hauschildt \& Ensman 1994). To produce light curves, the latter work also used the temperature structure prescribed by the one-group radiation-hydro results. In the present work we attempt to solve the problem of a scattering dominated supernova envelope doing all calculations time-dependently. We calculate the temperature structure self-consistently, and we do not make assumptions on the radiative equilibrium which is strongly violated during the shock breakout.

We analyze the light curve of SN 1987A with a multi-group radiation hydrodynamics code called Stella (Blinnikov \& Bartunov 1993, Blinnikov et al. 1998). The calculated broad band $(U B V, I U E \mathrm{UV})$ and bolometric light curves are compared with observations for the first $\sim 4$ months after core collapse. From this we update the constraints on model parameters such as the explosion energy and the extent of mixing.

We discuss the uncertainties in our predictions due to NLTE effects (which are not included in our modeling) by comparing with NLTE atmospheric calculations done by other authors. We believe that those uncertainties can be bracketed for the first months of SN 1987A light by two extreme assumptions on the scattering of photons in spectral lines. It is found that the predictions

for the first hours and days are fairly insensitive to the assumptions on spectral lines, because 
of the overwhelming dominance of electron scattering. So, for those epochs we produce reliable predictions for the soft X-ray/extreme UV flash of the supernova.

\section{Radiation Hydrodynamics}

Earlier modeling of the light curve of SN 1987A has adopted the following types of numerical approaches:

1. Equilibrium-diffusion radiation hydrodynamics, with a flux-limiter to ensure a smooth transition from diffusion to free-streaming regimes (Shigeyama et al. 1987; Arnett 1987, 1988; Grasberg et al. 1987; Woosley 1988; Utrobin 1993). Here equilibrium means one-temperature approximation where the radiation and gas have the same temperature. Local thermodynamical equilibrium (LTE) is assumed, and black body spectra are used to obtain monochromatic broad-band light curves.

2. Non-equilibrium, one-energy group (gray) radiation hydrodynamics, where equilibrium between the gas and the radiation is no longer assumed (i.e., two-temperature transfer). LTE is assumed and a diluted black body approximation is used for the monochromatic light curves (Ensman \& Burrows 1992; Mair et al. 1992).

3. Multi-energy group (non-gray) atmospheric codes combined with gray radiation hydrodynamics. Here the atmospheric structures, i.e., the distributions of temperature, density, and velocity at $\tau_{\text {sc }} \lesssim 100$ are obtained from the gray hydro code described above (Höflich 1991; Hauschildt \& Ensman 1994) and the emerging spectra are computed taking into account NLTE effects.

4. Full multi-energy group radiation hydrodynamics. This is the approach in our study, and the code we have used is called STELLA (Blinnikov \& Bartunov 1993; Blinnikov et al. 1998); a detailed description of new technical features of the code is given in Sorokina, Blinnikov, \& Nomoto (1999). LTE for ionization and atomic level populations is assumed.

STELLA solves the time-dependent equations for the angular moments of intensity averaged over fixed frequency bands, using up to 300 zones for the Lagrangean coordinate and up to 100 frequency bins (i.e., energy groups). This high number of frequency groups allows one to have a reasonably accurate representation of non-equilibrium continuum radiation. There is no need to ascribe any temperature to the radiation: the photon energy distribution can be quite arbitrary.

The coupling of multi-group radiative transfer with hydrodynamics means that we can obtain the color temperature in a self-consistent calculation, and that no additional estimates of thermalization depth as in the one-energy group model of Ensman \& Burrows (1992) are needed. Variable Eddington factors are computed, which fully take into account scattering and redshifts for 
each frequency group in each mass zone. The gamma-ray transfer is calculated using a one-group approximation for the non-local deposition of the energy of radioactive nuclei. Here we follow Swartz, Sutherland, \& Harkness (1995; see also Jeffery 1998), and we only use a purely absorptive opacity. This should be a good approximation.

In the equation of state, LTE ionizations and recombinations are taken into account. The effect of line opacity is treated as an expansion opacity according to the prescription of Eastman \& Pinto (1993; see also Blinnikov et al. 1998). Their approach is different from that of Shigeyama \& Nomoto (1990) who used Rosseland mean opacities for scattering and absorption processes, where the line opacities were assumed to be 0.009 and $0.01 \mathrm{~cm}^{2} \mathrm{~g}^{-1}$ for helium and heavier elements, respectively (Los Alamos opacity library). Our opacities are also different from those in other equilibrium-diffusion or one-group radiation hydro models, since instead of one single energy-averaged opacity we need opacities for all our energy groups.

\section{Models}

We have studied two progenitor models in some detail: the evolutionary model of Nomoto \& Hashimoto (1988) and Saio, Nomoto, \& Kato (1988b) (see also Shigeyama et al. 1988, Shigeyama \& Nomoto 1990, Yamaoka et al. 1991; Saio, Kato, \& Nomoto 1988a), and the non-evolutionary model of Utrobin (1993). Here we concentrate on the model studied by Nomoto and co-workers. The results for Utrobin's model (which gives one of the best fits to the bolometric light of SN 1987 A among the equilibrium diffusion models) are presented elsewhere.

In the evolutionary model, a star with the initial mass $M_{\mathrm{ms}}=23 M_{\odot}$ and low metallicity $Z$ $=0.005$ is evolved from the main-sequence and onwards, with the Schwarzschild criterion applied for convection. During the evolution from blue to red, the stellar mass decreases to $16.3 M_{\odot}$ and a helium core of $M_{\text {core }}=6.7 M_{\odot}$ is formed. During the red phase, $0.7 M_{\odot}$ of helium is mixed out into the hydrogen-rich envelope yielding $M_{\text {core }}=6.0 M_{\odot}$ and $M_{\text {env }}=10.3 M_{\odot}$. The dredge up of the helium enhances the surface helium abundance to $Y_{\text {surf }}=0.43$, which is large enough to move the star in HR-diagram from the red to the location of $\mathrm{Sk}-69^{\circ} 202$ in the blue. The resultant luminosity and radius are $L_{0}=1.3 \times 10^{5} L_{\odot}$ and $R_{0}=48.5 R_{\odot}$, respectively. Because the radius of the progenitor is not well constrained in the evolutionary model, we have also constructed a hydrostatic envelope model with a radius of $R_{0}=40 R_{\odot}$ and of $R_{0}=58 R_{\odot}$ which are fitted to the evolved $6 M_{\odot}$ He core. The $6 M_{\odot}$ He core was evolved through the iron core collapse (Nomoto \& Hashimoto 1988). The resultant explosion and explosive nucleosynthesis were calculated as in Hashimoto, Nomoto, \& Shigeyama (1989) and Thielemann, Hashimoto, \& Nomoto (1990). We have assumed in all our models (i.e., irrespective of the explosion energy) that the mass cut is located at $M_{\mathrm{r}} \equiv M_{\mathrm{c}}=1.6 M_{\odot}$, so that the mass of the ejecta is $M_{\mathrm{ej}}=14.7 M_{\odot}$. Following Shigeyama \& Nomoto (1990) we denote our standard model 14E1. Various suffixes have been added to distinguish between different models which have different explosion energies and initial radii, as well as other different physical properties. 
For our multi-group computation it is inconvenient to use the initial evolutionary 14E1 model directly. Instead, the star was constructed in hydrostatic equilibrium in the same way as was done in Blinnikov et al. (1998) for SN 1993J: as we need a much finer zoning in the outer layers in our calculations than was used in Nomoto \& Hashimoto (1988) and Saio et al. (1988b), a remap of the original model was done onto another grid. We assume that at the outer boundary (i.e., at $m=M, M$ being the total mass of the star) the material pressure vanishes, $p=0$, and that there is no radiation coming from the outside. The density structure found in this way is shown in Figure 1. The density of the free blue wind is only $\sim 10^{-16} \mathrm{~g} \mathrm{~cm}^{-3}$ when scaled as $r^{-2}$ from $10^{17} \mathrm{~cm}$ and inward to $3 \times 10^{12} \mathrm{~cm}$ (Lundqvist 1999). This falls outside the plot in Figure 1 .

As the shock wave propagates through the star, the interfaces where the composition changes suddenly from $\mathrm{C}+\mathrm{O}$ dominated to helium dominated, and then further out to hydrogen-dominated, are strongly Rayleigh-Taylor unstable. This induces mixing of the material before the shock breakout at the surface (e.g., Bandiera 1984, Ebisuzaki, Shigeyama, \& Nomoto 1989, Arnett et al. 1989b, Benz \& Thielemann 1990, Hachisu et al. 1990, 1991, Müller, Fryxell, \& Arnett 1991, Basko 1994). Particularly important for the light curve behavior is the mixing of hydrogen down to the central region. Mixing of ${ }^{56} \mathrm{Ni}$ into the hydrogen-rich envelope also affects the light curve, and is decisive for spectral (Utrobin, Chugai, \& Andronova 1995) and X-ray observations. The mixed abundance distribution in Figure 3 is inferred from the comparison of the X-ray and $\gamma$-ray light curves and spectra with observations (Kumagai et al. 1989). The outermost mixed ${ }^{56} \mathrm{Ni}$ corresponds to the velocity $\sim 4000 / \mathrm{kms}$ in the best fitting model of the light curve (see Fig. 20 below). The mixing is artificial, since $2 \mathrm{D}$ modeling failed to distribute ${ }^{56} \mathrm{Ni}$ further out than $\sim 2500$ /kms (Hachisu et al. . 1990, 1991). The density profile was only marginally changed by the mixing.

We have tested explosion energies in the range $E=(0.7-1.5) \times 10^{51}$ ergs and named the runs 14E0.7, 14E1, 14E1.3, ..., etc., where '14' denotes the ejecta mass in solar units, see Table 11. All models in the runs labeled without the suffix ' $U$ ' have the mixed composition shown in Figure 3 already prior to the model explosion. Each model was exploded by the deposition of heat energy in a layer of mass $\sim 0.03 M_{\odot}$ outside of $1.6 M_{\odot}$. Since stella does not include nuclear burning, preservation of the same mixed composition in the ejecta is assured. The explosion energies given above refers to the asymptotic kinetic energy of ejecta. The heat energy injected for a simulated explosion is higher by $\sim 0.7 \times 10^{51}$ ergs, i.e., by the gravitational binding energy for the presupernova model. A small fraction $\left(\sim 7 \times 10^{48} \mathrm{ergs}\right)$ goes to the photon energy which is radiated away. For other models, $14 \mathrm{E} 1 \mathrm{U}$, and $14 \mathrm{E} 1.2 \mathrm{U}$, the unmixed composition in Figure 2 was used, and in 14E1A, we treated the opacity as pure absorption for the same total extinction (which, however, is often dominated by scattering in reality). For the latter model the entry for "forced $\chi_{\text {abs }}$ " is "yes", i.e., $\chi_{\text {abs }}$ was artificially set equal to the total extinction. The suffix ' $\mathrm{H}$ ' denotes the model having the same density structure as the standard one, 14E1, but the abundance of hydrogen in the outer layers is enhanced to the solar value. The suffix ' $R$ ' is added for the models with non-standard initial radius (see Table 1). Some other models from the Table 1 
are discussed below.

\section{Hydrodynamics and Shock Breakout}

The shock wave arrives at the surface of the star at time $t_{\text {prop }}$, which for different values of the initial radius $R_{0}$, the ejected mass $M_{\mathrm{ej}}$, and explosion energy $E$, could be approximated by:

$$
t_{\text {prop }} \approx 1.6\left(\frac{R_{0}}{50 R_{\odot}}\right)\left[\left(\frac{M_{\mathrm{ej}}}{10 M_{\odot}}\right) /\left(\frac{E}{1 \times 10^{51} \mathrm{erg}}\right)\right]^{\frac{1}{2}} \text { hours }
$$

This expression is consistent with Shigeyama et al. (1987). We compare the estimate (1) with computations in Figure 4. The change in the velocity profile in Figure 6 shows how the materials are accelerated near the shock breakout until they reach homologous expansion. The maximum velocity at the outer edge is $\sim 32,500 \mathrm{~km} \mathrm{~s}^{-1}$ for $14 \mathrm{E} 1$ (which is somewhat lower than for $14 \mathrm{E} 1.3$ in Figure 6). Further acceleration is limited by the inefficiency of the radiative precursor of the shock (see a semi-analytic approach reviewed by Nadyozhin 1994; see also Imshennik \& Nadyozhin 1988). We will discuss the influence of various assumptions on the maximum velocity and other details of shock breakout elsewhere. Here we just note that velocities of the order $\sim(3-4) \times 10^{4} \mathrm{~km}$ $\mathrm{s}^{-1}$ are in very good agreement with the value found from the absorption feature of $\mathrm{Mg}$ II $\lambda 2800$ in the early IUE spectra (Pun et al. 1995). This has important implications for our understanding of the density structure of the circumstellar medium of the supernova (Chevalier 1999; Lundqvist 1999). More specifically, it indicates that the density of the blue supergiant wind must have been very low, corresponding to a mass loss rate of only $\lesssim 10^{-8} M_{\odot} \mathrm{yr}^{-1}$.

The density distribution in the outer part is well approximated by a power law $r^{-8.6}$ as found in Shigeyama \& Nomoto (1990), but the very outermost layers are much steeper (see Fig. 5). In between there is a dense shell, which was also found in non-equilibrium radiation hydrodynamic modeling (Blinnikov \& Nadyozhin 1991, Blinnikov, Nadyozhin, \& Bartunov 1991, Ensman \&

Burrows 1992), but missed in the equilibrium diffusion modeling. Note that the density in the central parts computed by STELLA is much smoother than shown in Figure 6 by Shigeyama \& Nomoto (1990).

\section{Early Light Curve}

After the shock breakout, the early light curve up to $t \sim 25$ days is powered by the diffusive release of the internal energy of the radiation field that is established by the shock wave. The bolometric light curve reaches its maximum luminosity of $L_{\mathrm{bol}} \sim(4-9) \times 10^{44} \mathrm{ergs} \mathrm{s}^{-1}$ (Table 2 and Fig. (7) immediately after shock breakout, and then drops rapidly by many orders of magnitude in $\sim 10$ days. 
The total energy radiated during the first two days amounts to $\sim 10^{47}$ ergs (Table 2), but most of the radiation is emitted in a soft X-ray/EUV burst, and was not observed. However, the burst had the important effect that it ionized the surrounding gas (e.g., Lundqvist \& Fransson 1996; Sonneborn et al. 1997; Lundqvist 1999). The resultant ionization of the circumstellar material is commented on briefly in $\S 7.1$, and will be compared with the observations in greater detail in Lundqvist, Blinnikov, \& Bartunov (1999a).

After the burst, the ejecta expand so rapidly that the interior temperature (both of the matter and radiation) decreases almost adiabatically as $r^{-1}$. As a result, the bolometric luminosity decreases sharply to $L_{\text {bol }} \sim(2-3) \times 10^{41} \mathrm{ergs} \mathrm{s}^{-1}$ to form a minimum of the bolometric light curve. Figure 9 demonstrates that the model with $E=10^{51}$ ergs (i.e, 14E1) gives the best agreement with the observed bolometric flux. Note that the agreement for 14E1 is much better than in Figure 7 of Shigeyama \& Nomoto 1990). (Note also the higher resolution in our figure than in the figure of Shigeyama \& Nomoto 1990). The light curves computed by the flux-limited diffusion in Shigeyama \& Nomoto (1990) produce a short plateau, $\sim 20$ days (see Figs. $16-19$ in Shigeyama \& Nomoto 1990). This is neither seen in the observations, nor in our models. The light curves computed here by the full radiative transport are in better agreement with observations for the same models (cf. Fig. 9) suggesting that the more accurate method of modeling gives results which are closer to reality.

The luminosity at this phase is lower than for typical Type II-P supernovae by a factor of 10 -20 . This is due to the small initial radius which leads to a low luminosity because a much larger fraction of the radiation field energy is lost by $P \mathrm{~d} V$ work than in ordinary Type II-P supernovae. This is well-known from early modeling of low-luminosity Type II-P supernovae (Imshennik \& Nadyozhin 1964; Chevalier 1976). For SN 1987A, the low luminosity was successfully demonstrated in the models of Shigeyama et al. (1987), Arnett (1987), Grasberg et al. (1987), Woosley (1988), Woosley, Pinto, \& Eastman (1988) and Utrobin (1993).

In Figure 7, we show the changes in the color temperature, $T_{\mathrm{c}}$, of the best blackbody fit to the flux, along with the effective temperature, $T_{\text {eff }}$, defined by the luminosity and the radius of last scattering $R$ through $L=4 \pi \sigma T_{\text {eff }}^{4} R^{2}$ (see Blinnikov et al. , 1998, for details of finding $R$ and from that $T_{\text {eff }}$ ). The maximum value of $T_{\mathrm{c}}$ is $\sim 1.2 \times 10^{6} \mathrm{~K}$ for model $14 \mathrm{E} 1$ (see Table 2), which is higher than the $7.6 \times 10^{5} \mathrm{~K}$ in Höflich's (1991) NLTE time-dependent calculation for 14E1.25, but similar to the temperatures found by Ensman \& Burrows (1992). Our results are in very good agreement with the estimates of Imshennik \& Nadyozhin $(1988,1989)$. We emphasize that our multi-group radiative transfer with hydrodynamics obtains this temperature in a self-consistent way, and no additional estimates of the thermalization depth (like in the one-group model of Ensman \& Burrows 1992) are needed. The large difference between color and effective temperatures is due to scattering (e.g., Sobolev 1980; Kolesov \& Sobolev 1982; Höflich 1991; Wagoner \& Montes 1993); the average energy of the photons is higher than that corresponding to the value of $T_{\text {eff }}$. The effect is a deficit of photons in the visual at maximum light compared to a model with forced absorption (Fig. 8). This effect continues throughout the first day after shock breakout. In Figure 10 we 
demonstrate the dominance of scattering in extinction for high temperatures.

Figure 11 shows $T_{\mathrm{c}}$ for the same three runs as in Figure 9, and one can see that $T_{\mathrm{c}}$ is rather insensitive to $E$ (except for the maximum $T_{\mathrm{c}}$ at shock breakout, as displayed in Table 2).

During the first day, the visual luminosity increases because the intensity peak is rapidly shifted into the optical due to the decreasing photospheric temperature. In order for the optical flare-up of the supernova to be seen at $6 \mathrm{mag}$ at $t=3 \mathrm{~h}$, the condition $t_{\text {prop }}<3 \mathrm{~h}$ (Eqn. [1]) should be satisfied, which requires a relatively large $E / M_{\mathrm{env}}$ and small $R_{0}$ (Shigeyama et al. 1987; Woosley et al. 1987). Also, the ejecta and the radiation field should have expanded rapidly so that the radiation temperature becomes lower and the radius of the photosphere becomes larger sufficiently fast. Therefore, the expansion velocity, and thus $E / M_{\text {env }}$, should be larger than certain values for a given initial radius.

Figure 12 shows the $V$ light curve for the model 14E1.21 with realistic scattering dominated opacity for the first hours of SN 1987A. Here, and for other models below, we have used a distance modulus of 18.5. (See Walker 1999, for a discussion on the uncertainty of this value.) We note that the $V$ curve exhibits an early local minimum which does not exist in equilibrium diffusion models (e.g., Woosley 1988, Arnett 1988; a small minimum was found also by Utrobin 1993). Höflich (1991) ascribed this to a non-LTE effect, and used the location in time of the minimum to constrain $E$. However, we too recover the local minimum despite our LTE approach. The reason for the minimum is that when the bolometric flux continues to fall (Fig. 9) after shock breakout, the bolometric correction overcomes the effect of the falling bolometric flux. The visual luminosity then increases because the intensity peak is rapidly shifted into the optical due to the decreasing photospheric temperature. Regardless of the exact cause of the difference between our and Höflich's results on the one hand, and equilibrium diffusion models on the other, the first maximum in $V$ in our models is just on the level of Jones' limit (Wampler et al. 1987) even for our 14E1.3 run, where the energy of explosion is $\sim 9 \times 10^{49}$ ergs higher (see Table 1) than the model 14E1.25 used by Höflich (1991; see also Höflich \& Wheeler 1999). The earlier appearance of the peak of the $V$-flux in our run is due to this higher energy.

Compared with observations, and especially the first $V$ observation by McNaught \& Zoltowski (1987; later revised by West \& McNaught 1992), the calculated $V$ curve in Figure 12 rises too slowly. In equilibrium diffusion modeling (e.g., Arnett 1988, Woosley 1988, Shigeyama \& Nomoto 1990) the $V$ flux rises much faster. The reason for this difference is that equilibrium diffusion models do not care whether the total extinction is absorptive or due to scattering. The spectra are assumed in those models to be blackbody with the temperature equal to $T_{\text {eff }}$. This is not a good approximation, since in reality the thermalization of photons takes place well below the surface of the last scattering resulting in $T_{\mathrm{c}} \gg T_{\text {eff }}$. In our run $14 \mathrm{E} 1 \mathrm{~A}$ we have assumed purely absorptive extinction, i.e., we force even the electron scattering to act as true absorption so that the thermalization of the photons occurs at an optical depth of order unity. We thereby reproduce the results obtained in equilibrium diffusion models. As a matter of fact, the $V$ curve in 14E1A 
rises even faster than was observed. (See Fig. 14 which shows the $U B V$ light curves for $14 \mathrm{E} 1$ and 14E1A for the first 5 days.) There is of course no physical reason why electron scattering should be treated as absorptive. From this it is clear that that an accurate treatment of scattering is crucial to constrain $E$. We will discuss this now.

Höflich (1991) was the first to show that non-equilibrium effects influence the $V$ light curve of SN 1987A drastically; the flux in $V$ is $\sim 2$ magnitudes lower for the first hours than in the equilibrium gray atmosphere case. Höflich (1991) tried a higher explosion energy than in 14E1 of Shigeyama \& Nomoto (1990) to compensate for this reduction of flux by adopting the model 14E1.25 computed in the flux-limited equilibrium diffusion approximation by Shigeyama \& Nomoto (1990). He then found a marginal agreement with the first data points of McNaught \& Zoltowski (1987). The same results have since been presented in Höflich \& Wheeler (1999). Compared with our $V$ light curve of 14E1.21 and 14E1.3 (see Figs. 12 and 13) the rise of the $V$ curve in Höflich's (1991) 14E1.25 model is significantly faster. To clarify this discrepancy, one should note that Höflich used the temperature structure of an equilibrium diffusion model, which can be appreciably different from that in our full transport models. We cannot say with certainty that this fully explains why our results and those of Höflich differ so much. It could be due to that the expansion opacity should be treated differently in the energy equation than is described in Höflich (1990; see Blinnikov 1996, 1997).

There are, of course, uncertainties also in our models. In particular, the role of NLTE effects needs to be further examined, but we cannot envisage that they are able to explain the big difference between us and Höflich (1991) for the first hours after shock breakout. At this epoch the extinction is totally dominated by electron scattering and spectral lines are not so important, see Figure 10. We see virtually no difference in our results for the first day when we treat the lines as fully absorptive, or as totally scattering dominated. The NLTE effects set in later, and are very important after a couple of weeks (cf. Baron et al. 1996). We are therefore confident that our results for this epoch are more accurate than Höflich's (1991).

We note that even Höflich's $V$-flux is lower than the observed after the new reductions by West \& McNaught (1992). A possible explanation to the discrepancy between models and observations is that the stars, used to calibrate the early plates of the supernova by West \& McNaught (1992), are too cool for an object with a color temperature of $T_{\mathrm{c}} \sim 10^{5} \mathrm{~K}$. (We find at $t=0.128$ day that $T_{\mathrm{c}}=1.14 \times 10^{5}, 1.05 \times 10^{5}, 9.8 \times 10^{4} \mathrm{~K}$ for the runs $14 \mathrm{E} 0.7,14 \mathrm{E} 1$, and 14E1.3, respectively.) The temperature at $t=0.128$ days decreases with increasing explosion energy because of the earlier emergence of the shock and the faster adiabatic cooling. It should be emphasized that our models are much hotter than equilibrium diffusion models (e.g., Arnet 1988, Woosley 1988, Utrobin 1993), and a comparison between observations and our results is therefore more sensitive to calibration errors than are equilibrium diffusion models. As we will see in $\S 6.1$ our models fit the early IUE observations well, and since these observations are less likely to have the same error, we cannot exclude calibration errors to be the cause of the mismatch in $V$. This would mean that the early true $V$ flux was lower than hitherto believed. We point out that 
we have changed various parameters in our models to try to make our $V$ flux increase faster and thereby fit the observations better. These experiments included enhancing the iron abundance, and varying the explosion energy and presupernova model within the limits allowed by the global light curve. However, none of these attempts reduced the discrepancy. (See, e.g., the results for two different initial radii in Fig. 13.)

\section{Before and After the Peak of the Light Curve}

After the minimum around day $\sim 10$, the observed bolometric light curve showed an almost exponential increase up to day $\sim 60$, and subsequently formed a plateau-like broad peak around day $\sim 100$. After a relatively rapid drop, the luminosity then declined slowly between $t=120-$ 400 days at the rate of ${ }^{56} \mathrm{Co}$-decay. The energy source responsible for the broad peak of the light curve, and the tail, is therefore without doubt the radioactive decay of ${ }^{56} \mathrm{Ni} \rightarrow{ }^{56} \mathrm{Co} \rightarrow{ }^{56} \mathrm{Fe}$. The total mass of initial ${ }^{56} \mathrm{Ni}$ in our models is $M_{\mathrm{Ni}} \approx 0.078 M_{\odot}$, which is the same mass as in the models of Shigeyama \& Nomoto (1990). The theoretical bolometric light curves for models 14E1, $14 \mathrm{E} 1 \mathrm{M}$, and 14E1U with a different extent of mixing are shown in Figure 19. It is clearly seen that the shape of the modeled light curve is strongly dependent on the distribution of hydrogen and ${ }^{56} \mathrm{Ni}$ in the ejecta, i.e., the amount of mixing that has occurred.

For the model with standard mixing (14E1 with ${ }^{56} \mathrm{Ni}$ mixed out to $v \sim 4000 \mathrm{~km} \mathrm{~s}^{-1}$, Fig. 20), there is significant heating of the outer layers due to radioactivity. The heating becomes noticeable in the light curve already at $\sim 10$ days, and then forms a smooth increase in the optical light curve up to the peak, as observed.

For the unmixed model 14E1U (Figs. 2, 21), the shape of its bolometric light curve starts to differ from that of 14E1 already at $\sim 10$ days. For the unmixed case, the increase in the luminosity due to radioactive heating is delayed until $t=35$ days, which causes a dip to appear in the light curve around day 30, and makes the light curve in the subsequent phase rise faster than in the mixed model. These properties are clearly incompatible with the observations.

For the case with intermediate mixing $(14 \mathrm{E} 1 \mathrm{M}),{ }^{56} \mathrm{Ni}$ is mixed out to $v \sim 2500 \mathrm{~km} \mathrm{~s}^{-1}$, while hydrogen is mixed as in the mixed model 14E1 (Fig. 21). Also in this model the appearance of radioactive heating occurs too late to be compatible with observations (see Fig. 19). This suggests that mixing of ${ }^{56} \mathrm{Ni}$ out to $v \sim 4000 \mathrm{~km} \mathrm{~s}^{-1}$ is needed. This conclusion is independent of the radiation transfer scheme used (Nomoto, Shigeyama, \& Hashimoto 1987; Woosley 1988; Shigeyama et al. 1988). The mixing out to large velocities is also supported by the redshifted feature at $\sim 3900 \mathrm{~km} \mathrm{~s}^{-1}$ observed by Haas et al. (1990; see also Utrobin, Chugai, \& Andronova 1995). We note, however, that Utrobin (1993) obtains a good bolometric light curve without ${ }^{56} \mathrm{Ni}$ mixing, and that Kozma \& Fransson (1998a,b) do not need to mix nickel out to more than $2000 \mathrm{~km} \mathrm{~s}^{-1}$ to model iron line profiles at late epochs. We postpone a more detailed discussion on mixing to a future paper. 
It is also interesting to compare the results of our non-equilibrium radiative transfer modeling by STELLA with the models in Figures 16 - 19 of Shigeyama \& Nomoto (1990). One striking difference, already noted above, is that the shape of the minimum near day 10 is reproduced much better with our non-equilibrium modeling. Another difference is that the shape of the light curve around maximum (at $\sim 3$ months) is smoother in models with realistic scattering opacity (see Fig. 29 below), than in models with forced absorption like 14E1A (see Fig. 32), and hence also in equilibrium diffusion models since the latter two types of model are closely related. These two models also show the same postmaximum sharp decline (cf. Utrobin 1993), which can be understood in terms of enhanced emission according to Kirchoff's law.

We note that the rising part of the light curve is modeled better with enhanced absorption in spectral lines than with scattering lines (see Fig. 32 for the bolometric luminosity, and Figs. 22, 23 for $U B V$ colors). This can perhaps be explained by NLTE effects. In particular, the effect of fluorescence could be important (see Baron et al. 1996, Li, McCray, \& Sunyaev 1993, Li \& McCray 1996, and references therein). This cannot be studied directly by STELLA, but, as found by Baron et al. (1996), forced absorption in spectral lines can reproduce some properties of the fluorescence as fluorescence is a form of thermalization; spectra of LTE models with absorptive lines are very similar to full NLTE spectra, while LTE models with scattering lines are far from reality (see also Eastman 1997; Blinnikov et al. 1998). This hints why the rising part is modeled well when we apply forced absorption in lines.

Another cause for the deviation of the rising part of the light curve from what was observed could be that the distribution of hydrogen is different from that in our model. This effect was investigated by Utrobin (1993). The hydrogen distribution affects the light curve because it determines how the hydrogen recombination front propagates into the ejecta (e.g., Nadyozhin 1994; Shigeyama \& Nomoto 1990). Because electron scattering is the main source of opacity, the opacity decreases sharply in the outward direction at the recombination front. This causes the photosphere to become associated with the recombination front. When the ejecta pass through this front their temperature quickly decreases to $\sim 5500 \mathrm{~K}$. This is seen as a change in the temperature profile in Figures 15 and 16. One can locate the photosphere in Figure 15 at the point where the radiation temperature starts to deviate from the material temperature appreciably. We note that the recombination front is much broader than that in Shigeyama \& Nomoto (1990) because of the large contribution of line opacity to the total opacity in our calculations. The effects of non-equilibrium transport are also important for the width of the recombination front.

As seen in Figures 15 and 17 the photosphere propagates inward in mass, while the material expands outward. For a certain period, the recombination front is almost stationary in radius (Figure [16). If $T_{\text {eff }}$ were constant, then this would result in an almost constant bolometric luminosity, i.e., in a perfect plateau of the light curve, where the duration of the plateau stage depends on how deep into the star hydrogen has been mixed. For a deeper mixing of hydrogen, the plateau lasts longer. When the photosphere enters into layers which lack sufficient amounts of hydrogen, the hydrogen recombination front disappears and the plateau phase is terminated. This 
is the case for typical Type II-P supernovae, where radioactive heating does not show up until the very end of the plateau stage (Eastman et al. 1994). SN 1987A is quite different in this respect, since radioactivity is important also at early epochs; the diffusion flux from the radioactive energy release starts to dominate over the diffusion flux from the recombination of hydrogen already at $\sim 6$ weeks after the explosion. This is clearly seen in Figure 17 (cf. Fig. 12 in Shigeyama \& Nomoto 1990, and Fig. 11 in Utrobin 1993).

The relation between the duration of the plateau phase and the depth of the hydrogen layer can be given more quantitatively. Suppose that hydrogen is mixed down to a shell where the expansion velocity of the hydrogen-rich layer is $v_{\mathrm{H}}$. Then this velocity is related to the observed quantities for the plateau phase as

$$
v_{\mathrm{H}} \approx 1300\left(\frac{L_{\mathrm{pl}}}{8.5 \times 10^{41} \operatorname{ergs~s}^{-1}}\right)^{1 / 2}\left(\frac{t_{\mathrm{pl}}}{100 \mathrm{~d}}\right)^{-1} \mathrm{~km} \mathrm{~s}^{-1}
$$

where $t_{\mathrm{pl}}$ is the time at the end of the plateau, $L_{\mathrm{pl}}=4 \pi \sigma T_{\mathrm{eff}}^{4} R^{2}$ the luminosity at $t \sim t_{\mathrm{pl}}$, and $T_{\text {eff }} \sim 5500 \mathrm{~K}$ because of the association of the photosphere with the hydrogen recombination front. The time $t$ is for the freely expanding ejecta, $t=R / v$. For example, if we take $L_{\mathrm{pl}}=2 \times 10^{41}$ ergs $\mathrm{s}^{-1}$, and $t_{\mathrm{pl}}=30$ days, as in the unmixed model $14 \mathrm{E} 1 \mathrm{U}$ (Fig. 19), we find from Eqn.(2) $v_{\mathrm{H}} \approx 2000 \mathrm{~km} \mathrm{~s}^{-1}$ which is in good agreement with Figure 21 .

In the standard model, $14 \mathrm{E} 1$, hydrogen is mixed down to $M_{\mathrm{r}} \approx 2 M_{\odot}$, i.e., only $\approx 0.4 M_{\odot}$ outside $M_{\mathrm{c}}$. The expansion velocity at that radius is only $\sim 1000 \mathrm{~km} \mathrm{~s}^{-1}$. If we adopt $t_{\mathrm{pl}} \sim 100$ days, Eqn. 2 gives $v_{\mathrm{H}} \sim 1300 \mathrm{~km} \mathrm{~s}^{-1}$ as is required from observations. Equation (2) thus appears to give a reasonable estimate of $v_{\mathrm{H}}$ when we put $t_{\mathrm{pl}}$ equal to the time when the second maximum ends. However, we caution that this result should not be overinterpreted. Equation (2) gives the photospheric velocity as long as $T_{\text {eff }}$ is $5500 \mathrm{~K}$, which is roughly the case for SN 1987A also for the second maximum. But the formula also assumes that the temperature is governed by the presence of hydrogen. This is not a unique statement, as the complicated thermal balance may settle around this temperature also without the presence of hydrogen. Even if hydrogen is mixed far into the core, the end of the second maximum is likely to give only limited information about the minimum velocity of hydrogen.

\subsection{Broadband fluxes}

Ideally, both spectra and $U B V$ colors should be obtained by full NLTE modeling (see arguments put forward by Eastman 1997). For SN 1987A spectral NLTE modeling has been made by several groups (see, e.g., Schmutz et al. 1990, Höflich 1990, Takeda 1991, Mazzali, Lucy, \& Butler 1992, Duschinger et al. 1995, Mazzali \& Chugai 1995, Höflich \& Wheeler 1999 and references therein), while NLTE modeling of colors are given in the literature only for the first days after the explosion (Eastman \& Kirshner 1989, Hauschildt \& Ensman 1994). Our approach cannot add to this NLTE modeling, but as discussed by, e.g., Höflich (1995, see also references 
therein), LTE modeling is useful even for such rapidly expanding objects as Type Ia SNe, and should therefore also give some insight to the physical conditions of SN 1987A. Inspired by this, we compare our LTE results with observations in Figure 22. The $B$ and $V$ light curves are in surprisingly good agreement with observations. Likewise, for the $U$ band the agreement is satisfactory for the first days, though at later epochs the modeled flux is too high. Nevertheless, the shape of the light curve qualitatively reproduces the observations.

There is also a qualitative agreement between our calculated fluxes and the observed fluxes in IUE bands (Pun et al. 1995) (see Figs. 24, 25). In particular, the agreement is good for the first 10 days for the SWP and the two LWP bands, while the modeled flux overshoots early for the LWP 3000 - $3300 \AA$ band, like it does for the modeled $U$ flux. Later, the modeled flux in the bands with the shortest wavelengths undershoots, while for the range $2500-3000 \AA$, the agreement is still fairly good. The modeled flux in the band with the longest wavelengths continues to be high for the first 100 days (Fig. 25).

The disagreement between modeled and observed UV fluxes after $\sim 100$ days is not surprising, because the LTE modeling at that epoch becomes quite unrealistic when there is no longer a true photosphere. For earlier epochs, there can be several causes for deviations. The early drop of the modeled fluxes in the shortest IUE bands could signal that the photosphere is somewhat hotter than in the 14E1 model; the flux here falls into the Wien part of the spectrum and it is exponentially sensitive to the temperature. It is harder to explain why the observed UV flux for $\lambda>3000 \AA$ and the flux in the $U$ band fall below what we predict. Within the LTE approach this could mean that the expansion opacity is not complete in the Eastman-Pinto (1993) approximation. The line list of Eastman \& Pinto includes $\sim 10^{5}$ lines, but they and Baron et al. (1996) have pointed out that it is also necessary to include millions of weaker lines (see also Höflich 1995), mostly of iron. Should the Eastman-Pinto list be sufficient, then one might think of enhancing the abundance of iron group elements in the outer layers of the supernova to increase the opacity. Our experiments with such an enhancement show that we can bring the $U$ flux in much better agreement with observations. There could also be other causes for our too strong UV flux, but it seems reasonable to assume that line opacities are somehow involved. This is highlighted by the good agreement between modeled and IUE fluxes in the $2500-3000 \AA$ range where there are relatively few spectral lines. This could perhaps indicate that the envelope is contaminated with heavy elements which could give a higher opacity in the UV.

In this context we note that Pun et al. (1995) do not cite Wagoner, Perez, \& Vasu (1991) correctly when they state that "the expansion opacities in the wavelength region 1000-4000 increase by a factor of more than 100 as the temperature of the atmosphere drops from 12,000 to $5000 \mathrm{~K}$ ". We point out that it is not the expansion opacity in Wagoner et al. (1991; see also Eastman \& Pinto 1993) which increases by this number. The correct statement is that the ratio of expansion opacity to electron scattering increases by a factor of $\gtrsim 100$. It does so because the electron scattering opacity drops drastically due to recombination as the temperature is lowered. 
We have not tried to include millions of weak lines in our models to check whether this can bring the UV observations and our LTE models in better agreement. If such an experiment would fail, and the metal content of the envelope is not unusually high (cf. above), then one has to consider NLTE effects already for a few days after the explosion. The main NLTE effect here could be the excitation of hydrogen from its second principal level, $n=2$, perhaps creating an optically thick Balmer continuum. From NLTE atmospheric calculations (see, e.g., Schmutz et al. 1990, Takeda 1991, Duschinger et al. 1995) we know that early overpopulation of $n=2$ is present in SN 1987A, though it is not sufficient to explain the observed absorption in the Balmer range, unless there is a direct excitation due to radioactivity. While it is certainly important to include nonthermal excitation in late spectra hydrogen (e.g., Xu et al. 1992; Kozma \& Fransson 1998b), the same effect operating at early times could be one more hint of extremely efficient outward mixing of radioactive material into the hydrogen-rich envelope. The possibility that hydrogen could be excited as a result of circumstellar interaction seems much more unlikely because of the very low density inferred for the circumstellar gas ( $\S 4$; Chevalier 1999; Lundqvist 1999).

\subsection{Parameters of the Photosphere}

We present in Figures 26-28 the comparison of our numerical results with the "photospheric" parameters found by observers. We put this in quotes since what is given by observers are not really the parameters of the photosphere, but the best blackbody fit temperature $T_{\text {obs }}$ (which is

higher than $T_{\text {eff }}$ ), and the radius, $R_{\mathrm{obs}}$, found from $L=4 \pi \sigma T_{\mathrm{obs}}^{4} R_{\mathrm{obs}}^{2}$. We emphasize that $R_{\mathrm{obs}}$ is substantially smaller than the radius of the true photosphere, $R_{\mathrm{ph}}$, especially at the earliest stages.

\section{Dependence on Model Parameters}

\subsection{Explosion Energy}

In the above discussion, the light curve has mainly been used to probe the internal abundance distribution. Figure 29 shows how the bolometric light curve depends on $E$. For a given ejecta mass and using the standard mixing, we can find constraints on $E$ from the light curve (Shigeyama et al. 1987, 1988; Nomoto et al. 1987, 1994; Woosley 1988; Woosley et al. 1988; Arnett \& Fu 1989; Imshennik \& Popov 1992). First, the luminosity near the minimum around day 10 (which corresponds to the early short plateau in the $V$ curve) is almost proportional to $E$ (Litvinova \& Nadezhin 1990, Popov 1993), thus providing an important constraint on $E$. Second, the time of the peak, $t_{\text {peak }} \sim 3$ months, depends on $E$. For larger $E$, i.e., faster expansion, the rise starts earlier because of earlier appearance of heating due to radioactivity; the decline after the peak is earlier because of a larger velocity of the ejecta which causes the diffusion time-scale to be shorter. The analytical treatment of this epoch is given in detail by Imshennik \& Popov (1992). We caution that it is not just $E$ that determines $t_{\text {peak }}$ (for a given hydrogen distribution), but the combination 
$E / M_{\mathrm{env}} \cdot t_{\text {peak }}$ is therefore mainly determined by $E / M_{\mathrm{env}}$ and the hydrogen distribution.

Figure 29 shows that both the first and the second parts of the light curve are reproduced well by $14 \mathrm{E} 1$ (see also Fig. 9). 14E1.3 is too bright near the minimum, while 14E0.7 is too dim near the minimum and evolves too slowly. Compared with the flux-limited diffusion model (Shigeyama \& Nomoto 1990) 14E1 evolves similarly, but in much better agreement with observations both near the minimum and near the peak when a more accurate radiative transfer scheme is used.

For the progenitor we have used in Figure 29 (i.e., the model with mixing and $M_{\text {env }}=10.3 M_{\odot}$ ), the best explosion energy in order to fit the observations is close to $1.1 \times 10^{51} \mathrm{ergs}$. Considering the uncertainties of the progenitor model in terms of mixing and envelope mass, we obtain best fits to the bolometric light curve for the explosion energies in the range $(0.85-1.35) \times 10^{51} \mathrm{ergs}$. As noted in Figure 9, the "true" bolometric is likely to be intermediate to the SAAO and CTIO/ESO results used in our fits (see Suntzeff \& Bouchet 1990). This allows for a $\pm 10 \%$ span in luminosity. However, a larger error in luminosity ( 15\%, Lundqvist et al. 1999b) is due to the still prevailing uncertainty in distance modulus to the supernova (e.g., Walker 1999), which gives a combined error of approximately $\pm 30 \%$ in absolute luminosity. The explosion energy should therefore be within the range $(0.8-1.4) \times 10^{51}$ ergs.

We note that $E$ is very similar to in the analytical diffusion models of Arnett \& Fu (1989) and Imshennik \& Popov (1992). (The initial theory of diffusion was developed by Arnett [1980, 1982] for Type Ia supernovae.) All these models use an eigenvalue formulation of the problem, which is not strictly correct (Blinnikov \& Popov 1993). However, the more correct, though more complicated, moving-boundary formulation produces results which agree rather well with the results of Imshennik \& Popov (1992; see Popov 1995). We therefore support the use of the results of Imshennik \& Popov (1992) to make reliable estimates of the supernova parameters from the near maximum light.

This rather limited range of energies we find is important for calculations of the ionization of the circumstellar gas (e.g., Lundqvist \& Fransson 1996; Lundqvist 1999). Until now these calculations have been based on the models 500 full1 and $500 f u l l 2$ of Ensman \& Burrows (1992). Qualitatively, the $500 \mathrm{full1}$ model is rather similar to the models in our preferred energy range. We will discuss this in detail in Lundqvist et al. (1999a), but from the analysis in Lundqvist \& Fransson (1996) we note immediately that the ionization of the outer rings is particularly sensitive to the spectrum of the burst.

\subsection{Radius of the Progenitor}

The above analysis has been based on a progenitor model with $R_{0}=48.5 R_{\odot}$. The uncertainty in the luminosity of the progenitor may imply that $R_{0}$ could be uncertain by $\sim 20 \%$ Woosley 1988, Saio et al. 1988b). Thus we have calculated the light curve models 14E1.26R, 14E1.34R, and 14E1.45R for $R_{0}=40 R_{\odot}$, and one model, 14E1.4R6, for $R_{0}=58 R_{\odot}$ (Table 11 and Figs. 30, 31). 
The early light curve for the first 2 days is not so different from the case of the standard $R_{0}=$ $48.5 R_{\odot}$, but $t_{\text {prop }}$ scales as $R_{0} / E^{1 / 2}$.

However, the light curve near the minimum (plateau in $V$ ) is dimmer for $R_{0}=40 R_{\odot}$ because the luminosity at that epoch is approximately proportional to $R_{0}$. As a result, for $R_{0}=40 R_{\odot}$, we need an explosion energy larger than $1.2 \times 10^{51} \mathrm{ergs}$ to put the light curve in agreement with the observations near the minimum (Fig. 30). But then the peak is too early. A larger radius, $R_{0}=$ $58 R_{\odot}$, shows an opposite trend. In this case, one needs a lower energy for the brightness at the minimum, but then the peak is too late.

\subsection{Hydrogen mass}

We have also made some runs for models where the hydrogen abundance in the outer layers was artificially raised to 0.7 (as is also assumed in, e.g., the non-evolutionary models by Utrobin 1993). For example, the model 14E1.25H (Table 1) has the same composition of metals as 14E1, but $\mathrm{H}$ is enhanced at the expense of He. So, the total mass of $\mathrm{H}$ is here $M_{\mathrm{H}} \sim 7 M_{\odot}$, while it was $M_{\mathrm{H}} \sim 5.5 M_{\odot}$ in the standard runs (14E1, 14E1.3 etc.). The hydrogen rich model $14 \mathrm{E} 1.25 \mathrm{H}$ evolves similarly to the standard runs 14E1.3 and 14E1.21 (Fig. 33). This explains the success of such models, as demonstrated by Utrobin (1993), but it is hard to justify a solar $\mathrm{H}$ abundance for SN 1987A from the evolutionary point of view, as well as in context of the abundances in the inner circumstellar ring (e.g., Lundqvist \& Fransson 1996).

\section{Conclusions}

In this paper we have described an extensive set of full radiation hydrodynamics calculations aimed to improving the modeling of the first few months of the light curve of SN 1987A. We have shown that the improved models can reproduce the light curve and suggest that proper handling of the radiation transfer is indeed decisive for the success of model fits.

The full multi-group radiation hydrodynamic modeling is more reliable mainly because the effects of scattering are treated self-consistently. Our findings are that:

1. the color temperatures and broad band photometry (and full continuum spectra) are predicted correctly for the first days of the supernova.

2. the shape of the modeled light curve, especially near the bolometric minimum at $\sim 10$ days, and during the broad peak at $\sim 100$ days is much better in the multi-group approach than in the equilibrium diffusion one.

3. the density profiles of the supernova at various epochs are smoother. 
Our code assumes LTE, but we have bracketed NLTE effects by extreme assumptions of the treatment of spectral lines (as either being scattering dominated, or fully absorptive). We find that the emission at shock break-out is not sensitive to those assumptions, which gives us confidence in our results. This is supported by a very good agreement with $I U E$ observations for the first days. For the first 100 days, best agreement is obtained when NLTE effects are mimicked by treating the line opacity as absorptive, following the prescription of Baron et al. (1996).

Looking at our results in greater detail, we find that the color temperature around shock breakout exceeds $10^{6} \mathrm{~K}$, which is higher than those obtained using a more approximate approach (e.g., Shigeyama \& Nomoto 1990), but not so different from the model 500 full1 of Ensman \& Burrows (1992). The large difference between color and effective temperatures persists for the first hours. This implies that the rise in the $V$ luminosity is slower than in equilibrium diffusion models (which was first noticed by Höflich 1991). Because of the overwhelming dominance of electron scattering during the first hours, the rise in the $V$ band is too slow in all of our models. This raises the question on the calibration of the first photometry data of SN 1987A (McNaught \& Zoltowski 1987; West \& McNaught 1992).

It is indicated by our light curve modeling that mixing of ${ }^{56} \mathrm{Ni}$ up to $v \sim 3000-4000 \mathrm{~km}$ $\mathrm{s}^{-1}$ could be needed, but our analysis is unlikely to supersede those of spectral analysis (e.g., Utrobin, Chugai, \& Andronova 1995, Kozma \& Fransson 1998a,b), modeling of early X-ray emission (e.g., Kumagai et al. 1989), or direct observations of infrared IR lines (Erickson et al. 1988).

We have improved on the constraining of $E$. The earlier flux-limited diffusion calculations (Shigeyama \& Nomoto 1990) provided a constraint on $E$ from both the pre-peak light curve and the plateau-like maximum light, concluding $E=(1.1 \pm 0.4) \times 10^{51} \mathrm{ergs}$. We find from our more detailed analysis that the best agreement with the observations is obtained for $E=(1.1 \pm 0.3) \times 10^{51}$ ergs. To arrive at this result we have assumed that the most likely range of $M_{\text {env }}$ is $M_{\text {env }}=7$ $10 M_{\odot}$ (Saio et al. 1988b), in order for models of the presupernova evolution to be consistent with the enhancement of N/C and N/O in the circumstellar matter (Lundqvist \& Fransson 1996). Knowing the energy with this accuracy, as well as having a detailed spectroscopic evolution from our models, we can constrain the ionization of the circumstellar gas much better than before. This will be discussed in Lundqvist et al. (1999a).

We are grateful to Ron Eastman, Stan Woosley, Wolfgang Hillebrandt, Vladimir Imshennik, Dmitriy Nadyozhin, Nikolai Chugai, Cecilia Kozma, Alexandra Kozyreva, Jason Pun and Victor Utrobin for discussions, and also Ron Eastman, Vlad Popolitov and Elena Sorokina for using their computer codes as part of ours.

Preliminary results of this work were reported at the "CTIO/ESO/LCO Workshop on SN 1987A: Ten years after" in the contributions by Lundqvist \& Sonneborn (1999) and Nomoto, Blinnikov, \& Iwamoto (1999). We would like to thank the participants of this workshop for 
stimulating discussions.

This work was supported in Russia by a grant from The International Science \& Technology Center 97-370, and by the Russian Basic Research Foundation grants RBRF 96-02-19756 and RBRF 96-02-17604. We are also grateful to grants from The Royal Swedish Academy of Sciences and The Wenner-Gren Center Foundation for Scientific Research. P.L. receives further support from The Swedish Natural Science Research Council and The Swedish Board of Space Research. The project has in part also been supported by the grant-in-Aid for Scientific Research (05242102, 06233101) and COE research (07CE2002) of the Ministry of the Education, Science, Culture, and Sports in Japan. 


\section{REFERENCES}

Arnett, W. D. 1980, ApJ, 237, 541

Arnett, W. D. 1982, ApJ, 253, 785

Arnett, W. D. 1987, ApJ, 319, 136

Arnett, W. D. 1988, ApJ, 331, 377

Arnett, W. D., Bahcall, J. N., Kirshner, R. P., \& Woosley, S. E. 1989a, ARAA, 27, 629

Arnett W. D., Fryxell B., Müller E., 1989b, ApJ, 341, L63

Arnett, W. D., \& Fu, A. 1989, ApJ, 340, 396

Bandiera, R. 1984, A\&A, 139, 368

Baron, E., Hauschildt, P. H., Nugent, P., \& Branch, D. 1996, MNRAS, 283, 297

Basko, M. 1994, ApJ, 425, 264

Benz, W. \& Thielemann, F.-K. 1990, ApJ, 348, L17

Blinnikov, S. I. 1996, Astron. Lett. J. Astron. Space Sci., 22, 92

Blinnikov, S. I. 1997, in Proc. NATO ASI, Thermonuclear Supernovae, ed. P. Ruiz-Lapuente et al. (Dordrecht: Kluwer), 589

Blinnikov, S. I., \& Bartunov, O. S. 1993, A\&A 273, 106

Blinnikov, S. I., Eastman, R., Bartunov, O. S., Popolitov, V. A., \& Woosley, S. E. 1998, ApJ, 496, 454

Blinnikov, S. I., \& Nadyozhin, D. K. 1991, in Supernovae, ed. S. E. Woosley (New York: Springer), 213

Blinnikov, S. I., \& Nadyozhin, D. K., \& Bartunov, O. S. 1991, in High-Energy Astrophysics. American and Soviet perspectives, ed. H. G. Lewin et al. (Washington: National Academy Press), 39

Blinnikov, S. I., \& Popov, D. V. 1993, A\&A, 274, 775

Catchpole, R. M. et al. 1987, MNRAS, 229, 15

Chevalier, R. A. 1976, ApJ, 208, 826

Chevalier, R. A. 1999, in Proc. CTIO/ESO/LCO Workshop, SN 1987A: Ten years after, ed. M. M. Phillips, N. B. Suntzeff, Astronomical Soc. of Pacific, in press 
Duschinger, M., Puls, J., Branch, D., Höflich, P., \& Gabler, A. 1995, A\&A, 297, 802

Eastman, R. G., 1997, in Proc. NATO ASI, Thermonuclear Supernovae, ed. P. Ruiz-Lapuente et al. (Dordrecht: Kluwer), 571

Eastman, R. G., Kirshner, R. P. 1989, ApJ, 347, 771

Eastman, R. G., \& Pinto, P. A. 1993, ApJ, 412, 731

Eastman, R. G., Woosley, S. E., Weaver, T. A., \& Pinto, P. A. 1994, ApJ, 430, 300

Ebisuzaki, T., Shigeyama, T., \& Nomoto, K. 1989, ApJ, 344, L65

Ensman, L., \& Burrows, A. 1992, ApJ,

Erickson, E. F., Haas, M. R., Colgan, S. W. J., Lord, S. D., Burton, M. G., Wolf, J., Hollenbach, D. J., Werner, M. 1988, ApJ, 330, L39

Grasberg, E. K., Imshennik, V. S., Nadezhin, D. K., \& Utrobin, V. P. 1987, Soviet Astronomy Letters, 13, 227

Haas, M. R., Colgan, S. W. J., Erickson, E. F., Lord, S. D., Burton, M. G., \& Hollenbach, D. J. 1990, ApJ, 360, 257

Hachisu, I., Matsuda, T., Nomoto, K., \& Shigeyama, T. 1990, ApJ, 358, L57

Hachisu, I., Matsuda, T., Nomoto, K., \&, Shigeyama, T. 1991, ApJ, 368, L27

Hamuy, M. et al. 1988, AJ, 96, 1864

Hashimoto, M., Nomoto, K., \& Shigeyama, T. 1989, A\&A, 210, L5

Hauschildt, P. H., \& Ensman, L. M. 1994, ApJ, 424, 905

Hillebrandt, W., \& Höflich, P. 1989, Reports on progress in Physics 52, 1421

Höflich, P. 1990, Dr. Rer. nat. habil., Ludwig-Maximilians-Universität, Munich (MPA 563)

Höflich, P. 1991, in Proc. ESO/EIPC Workshop, Supernova 1987A and Other Supernovae, ed. I. J. Danziger \& K. Kjär (Garching: ESO), 387

Höflich, P. 1995, ApJ, 443, 89

Höflich, P., \& Wheeler, C. 1999, in Proc. CTIO/ESO/LCO Workshop, SN 1987A: Ten years after, ed. M. M. Phillips, N. B. Suntzeff, Astronomical Soc. of Pacific, in press (astro-ph/9705132)

Imshennik, V. S., \& Nadyozhin, D. K. 1964, AZh, 41, 829 (SvA AJ 8, 664)

Imshennik, V. S., \& Nadyozhin, D. K. 1988, Sov. Astron. Lett., 14, 449 
Imshennik, V. S., \& Nadyozhin, D. K. 1989, Sov. Science: Ap. Space Phys. Rev., 8, 1

Imshennik, V. S., \& Popov, D. V. 1992, AZh, 69, 497

Imshennik, V. S., \& Utrobin, V.P. 1977, SvA Letters, 3, 34

Jeffery, D. J. 1998, in Proc. 2nd Oak Ridge Symposium on Atomic and Nuclear Astrophysics, Stellar Explosions and Galactic Chemical Evolution, ed. A. Mezzacappa, Institute of Physics Publishing, 687 astro-ph/9811356

Kolesov, A. K., \& Sobolev, V. V. 1982, Astron Zh. 59, 417

Kozma, C., \& Fransson, C. 1998a, ApJ, 496, 946

Kozma, C., \& Fransson, C. 1998b, ApJ, 497, 431

Kumagai, S., Shigeyama, T., Nomoto, K., Itoh, M., Nishimura, J., \& Tsuruta, S. 1989, ApJ, 345, 412

Li, H., \& McCray, R. 1996, ApJ, 456, 370

Li, H., McCray, R., \& Sunyaev, R. A. 1993, ApJ, 419, 824

Litvinova, I. Y. \& Nadezhin, D. K. 1990, Soviet Astronomy Letters, 16, 29

Lundqvist, P. 1999, ApJ, 511, 389

Lundqvist, P., Blinnikov, S. I., \& Bartunov, O. S. 1999a, in preparation

Lundqvist, P., \& Fransson, C. 1996, ApJ, 464, 924

Lundqvist, P., Sollerman, J., Kozma, C., Larsson, B., Spyromilio, J., Crotts, A. P. S., Danziger, J., \& Kunze, D. 1999b, A\&A, 347, 500

Lundqvist, P., \& Sonneborn, G. 1999, in Proc. CTIO/ESO/LCO Workshop, SN 1987A: Ten years after, ed. M. M. Phillips, N. B. Suntzeff, Astronomical Soc. of Pacific, in press (astro-ph/9707144)

Mair, G., Hillebrandt, W., Höflich, P., \& Dorfi, A. 1992, A\&A, 266, 266

Mazzali, P. A., \& Chugai, N. N. 1995, A\&A, 303, 118

Mazzali, P. A., Lucy, L. B., \& Butler, K. 1992, A\&A, 258, 399

McNaught, R. H., \& Zoltowski, F. 1987, IAU Circ. 4389

Müller, E., Fryxell, B., \& Arnett, D. 1991, A\&A, 251, 505

Nadyozhin, D. K. 1994, in Supernovae, ed. S. A. Bludman et al. (Elsevier Science: Amsterdam), 569 
Nomoto, K., Blinnikov, S. I., \& Iwamoto, K. 1999, in Proc. CTIO/ESO/LCO Workshop, SN 1987A: Ten years after, ed. M. M. Phillips, N. B. Suntzeff, Astronomical Soc. of Pacific, in press

Nomoto, K., \& Hashimoto, M. 1988, Physics Report, 163, 13

Nomoto, K., Shigeyama, T., \& Hashimoto, M. 1987, in Proc. ESO Workshop on the SN 1987A, ed. I. J. Danziger (Garching: ESO), 325

Nomoto, K., Yamaoka, H., Shigeyama, T., Kumagai, S., \& Tsujimoto, T. 1994, in Supernovae, ed. S. A. Bludman et al. (Elsevier Science: Amsterdam), 199

Phillips, M. M. et al. 1988, AJ, 95, 1087

Pinto, P. A., \& Eastman, R. G. 1996 astro-ph/9611195)

Pizzochero, P. 1990, ApJ, 354, 333

Popov, D. V. 1993, ApJ, 414, 712

Popov, D. V. 1995, Pis'ma AZh, 21, 682 (Astronomy Letters, 21, 610)

Pun, C. S. J., et al. 1995, ApJS, 99, 223

Saio, H., Kato, M., \&, Nomoto, K. 1988a, ApJ, 331, 388

Saio, H., Nomoto, K., \& Kato, M. 1988b, Nature, 334, 508

Schmutz, W., Abbott, D. C., Russell, R. S., Hamann, W. -R., \& Wessolowski, U. 1990, ApJ, 355, 255

Shelton, I.K., \& Lapasset, E. 1993, AJ, 105, 1892

Shigeyama, T., \& Nomoto, K. 1990, ApJ, 360, 242

Shigeyama, T., Nomoto, K., \& Hashimoto, M. 1988, A\&A, 196, 141

Shigeyama, T., Nomoto, K., Hashimoto, M., \& Sugimoto, D. 1987, Nature, 328, 320

Sobolev, V. V. 1980, Astrofizika, 16, 695

Sonneborn, G., , Fransson, C., Lundqvist, P., Cassatella, A., Gilmozzi, R., Kirshner, R. P., Panagia, N., \& Wamsteker, W. 1997, ApJ, 477, 848

Sorokina, E. I., Blinnikov, S. I., \& Nomoto, K. 1999, in preparation

Suntzeff, N. B., \& Bouchet, P. 1990, AJ, 99, 650

Swartz, D. A., Sutherland, P. G., \& Harkness, R. P. 1995, ApJ, 446, 766 
Takeda, Y. 1991, A\&A, 245, 182

Thielemann, F.-K., Hashimoto, M., \& Nomoto, K. 1990, ApJ, 349, 222

Utrobin, V. 1993, A\&A, 270, 249

Utrobin, V. P., Chugai, N. N., \& Andronova, A. A. 1995, A\&A, 295, 129

Wagoner, R. V., \& Montes, M. 1993, Phys. Rep. 227, 205

Wagoner, R. V., Perez, C. A., \& Vasu, M. 1991, ApJ, 377, 639

Walker, A. R. 1999, in Post Hipparcos Cosmic Candles, ed. F. Caputo \& A. Heck, (Kluwer: Dordrecht), in press (astro-ph/9808336)

Wampler, E. J. 1988, in SN 1987A, One year later, ed. M. Greco (Editions Frontiers), 17

Wampler, E. J., Lucy, L. B., Truran, J. W., Höflich, P.; Hillebrandt, W. 1987, A\&A, 182, L51

West, R. M., \& McNaught, R. H. 1992, A\&A, 256, 447

Woosley, S. E. 1988, ApJ, 330, 218

Woosley, S. E., Pinto, P. A., \& Ensman, L. 1988, ApJ, 324, 466

Woosley, S. E., Pinto, P. A., Martin, P. G., \& Weaver, T. A. 1987, ApJ, 318, 664

Xu, Y., McCray, R., Oliva, E., \& Randich, S. 1992, ApJ, 386, 181

Yamaoka, H., Saio, H., Nomoto, K., \& Kato, M. 1991, in IAU Symp. 143, Wolf Rayet Stars and Interrelations with Other Massive Stars in Galaxies, ed. K. Van der Hucht \& B. Hidayat, (Kluwer), 571 
Fig. 1.- Density as a function of the interior mass, $M_{\mathrm{r}}$, and of the radius $r$ in our remap of the presupernova model 14E1 (compare with Fig. 1 in Shigeyama \& Nomoto 1990). Mass cut is at $M_{\mathrm{c}}=1.6 M_{\odot}$.

Fig. 2.- Composition as a function of interior mass, $M_{\mathrm{r}}$, for the most abundant elements in the unmixed presupernova model from Nomoto \& Hashimoto (1988) used for runs 14E1U and 14E1.2U. Mass cut is at $M_{\mathrm{c}}=1.6 M_{\odot}$.

Fig. 3.- Abundance distribution as a function of enclosed mass for the ejecta in model 14E1 with mixing.

Fig. 4.- The time for the shock to reach the surface of the star according to Eqn 1. versus the computed time for the models in Table 2. Dashed line shows direct proportionality between the two times.

Fig. 5.- Density profile against radius at $t=101$ days for model $14 \mathrm{E} 1$.

Fig. 6.- Changes in the velocity profile near shock breakout for model 14E1.3. The figure shows how the outermost ejecta are accelerated to homologous expansion.

Fig. 7.- Very early bolometric light curve, and color and effective temperatures for the run $14 \mathrm{E} 1$. Realistic, scattering dominated opacity has been assumed. Solid line shows the temperature of the best blackbody fit to the flux (color temperature). Dashed line shows the effective temperature defined by the luminosity and the radius of last scattering.

Fig. 8.- Spectral flux in observer's frame at first maximum light for models 14E1 (i.e., with realistic scattering; dashed line) and 14E1A (i.e., with forced absorption; solid line).

Fig. 9. - Early bolometric luminosity for runs 14E1.3 (dashed), 14E1 (solid), 14E0.7 (shortdashed). Observations are from data obtained at SAAO (squares, Catchpole et al. 1987) and CTIO (crosses Hamuy et al. 1988). Note that we in figures showing the bolometric light curve have chosen to show both the SAAO and CTIO/ESO results. The difference between the two photometric systems results in a maximum difference in bolometric luminosity of $\sim 1.2$ As argued in Suntzeff \& Bouchet (1990), the "true" result is likely to be intermediate to the SAAO and CTIO/ESO results. (See also §7.1.)

Fig. 10. - Absorption ( $\alpha$, solid) and scattering ( $\sigma$, dotted) opacity as a function of wavelength for three different temperatures. Density is the same in all plots, $\rho=10^{-10} \mathrm{~g} \mathrm{~cm}^{-3}$. This density is typical for the photospheric layer at the peak luminosity at shock breakout. Note the dominance of scattering for $T \gtrsim 3 \times 10^{4} \mathrm{~K}$.

Fig. 11. - Same as Figure 9 but for the color temperature $T_{\mathrm{c}}$. $T_{\mathrm{c}}$ was derived from a best-fitting blackbody to the modeled spectrum. 
Fig. 12.- Apparent $V$ magnitude in observer's frame for model 14E1.21. A reddening by $E_{B-V}=0.15$ (Wampler, 1988) was applied, and the distance modulus was assumed to be 18.5. Jones' limit is shown by inverted Y. "1987" marks $\mathrm{m}=6.36 \pm 0.11$ measured by McNaught \& Zoltowski (1987), and "1992" is $\mathrm{m}=5.94 \pm 0.10$ found by West \& McNaught (1992). The filled circles are the NLTE predictions by Höflich (1990, 1991), who took the temperature structure from the 14E1.25 model computed in the flux-limited equilibrium diffusion approximation by Shigeyama \& Nomoto (1990).

Fig. 13. $-V$ light curve for the first day for two values of the initial radius $R_{0}=48.5 R_{\odot}$ and $40 R_{\odot}$ (i.e., models $14 \mathrm{E} 1.3$ and $14 \mathrm{E} 1.34 \mathrm{R}$, respectively.) The 'old' reductions of the observations are given according to the compilation in Arnett (1988) and the 'new' reductions are from West \& McNaught (1992) and Shelton \& Lapasset (1993).

Fig. 14. - Early $U B V$ fluxes in observer's frame for $14 \mathrm{E} 1$. A reddening by $E_{B-V}=0.15$ and a distance modulus of 18.5 have been applied. The uppermost curve is the $V$ flux for 14E1A (forced absorption). Jones' limit is shown by inverted Y.

Fig. 15. - Change in the temperature profile against $M_{\mathrm{r}}$ for 14E1. Solid and dotted lines show gas and radiation temperatures, respectively. The attached numbers show the time in days after the explosion. Note that the time here is the comoving time, and not the retarded, as in graphs for observations. The hydrogen recombination front is rather broad and propagates inward in $M_{\mathrm{r}}$.

Fig. 16. - Same as Figure 15 but only for the material temperature against radius $r$.

Fig. 17. - Same as Figure 15 but for the comoving luminosity $L$. The drop in $L$ in the outer layers is explained by the fast motion of those layers (compare to the $L(r)$ graph in Fig. 17). Another reason for the drop in $L$ is the effect of retardation. For $t=105$ days the outer layers 'remember' the higher luminosity some time ago.

Fig. 18. - Comoving luminosity $L$ against radius $r$ in linear scale for days 59 and 105 .

Fig. 19.- Bolometric light curves for 14E1 (with mixed composition), 14E1M (mild mixing of ${ }^{56} \mathrm{Ni}$ ) and the unmixed model 14E1U. Squares are the data of Catchpole et al. (1987), crosses Hamuy et al. (1988).

Fig. 20.- Abundance distribution as a function of expansion velocity for the model 14E1 with mixing.

Fig. 21. - Distribution of $\mathrm{H}$ and ${ }^{56} \mathrm{Ni}$ as a function of expansion velocity in the unmixed model $14 \mathrm{E} 1 \mathrm{U}$ (solid and short-dashed lines) and in 14E1M (mild mixing of ${ }^{56} \mathrm{Ni}$, dashed and dotted lines).

Fig. 22. $-U B V$ magnitudes in observer's frame for model 14E1. A reddening of $E_{B-V}=0.15$ and a distance modulus of 18.5 have been applied. 
Fig. 23. - Same as in Figure 22 but for the run 14E1S in which the line opacity is scattering dominated.

Fig. 24. - Predicted fluxes in IUE bands for model 14E1 during the first 10 days (solid lines). The fluxes are in observer's frame, and the distance modulus has been set to 18.5. The observational data of Pun et al. (1995) are dereddened.

Fig. 25.- Same as in Figure 24 but for the first 100 days.

Fig. 26. - Best blackbody fit temperature (solid line), and effective temperature (dotted) for 14E1. Squares are data of Catchpole et al. (1987), crosses - Hamuy et al. (1988).

Fig. 27.- Radius of the "photosphere", $R_{\text {obs }}$, found from the blackbody fit temperature for $14 \mathrm{E} 1$ (solid line). Dashed line shows the radius $R_{2 / 3}$, where the optical depth in the continuum is $\approx 2 / 3$ at $\lambda \approx 5000 \AA$. Squares are data of Catchpole et al. (1987), crosses - Hamuy et al. (1988) for $R_{\mathrm{obs}}$.

Fig. 28. - Matter velocity at the "photosphere", $R_{\text {obs }} / t$, and at the optical depth $2 / 3, R_{2 / 3} / t$ (dashed), for 14E1. Squares are data of Catchpole et al. (1987), crosses - Hamuy et al. (1988). Circles are the values of $v$ found from Fe II $\lambda 5169$ by Phillips et al. 1988.

Fig. 29.- Bolometric light curves for different explosion energies: 14E1.3 (dashed), 14E1 (solid), 14E0.7 (short-dashed) for the mixed composition in Figure 3. Squares are data of Catchpole et al. (1987), crosses - Hamuy et al. (1988).

Fig. 30.- Bolometric light curves for $R=40 R_{\odot}$ for runs $14 \mathrm{E} 1.26 \mathrm{R}$ and $14 \mathrm{E} 1.45 \mathrm{R}$, which both have mixed composition.

Fig. 31. - Bolometric light curves for $R=40 R_{\odot}$ for run $14 \mathrm{E} 1.45 \mathrm{R}$, and $R=58 R_{\odot}$ for run 14E1.4R6.

Fig. 32. - Bolometric magnitude for model 14E1A (forced absorption instead of scattering, solid) and 14E1S (scattering lines, dashed). Squares are data of Catchpole et al. (1987), crosses - Hamuy et al. (1988)

Fig. 33. - Bolometric light curves for the hydrogen rich model $14 \mathrm{E} 1.25 \mathrm{H}$, and the standard models $14 \mathrm{E} 1$ and $14 \mathrm{E} 1.21$. 
Table 1: Runs

\begin{tabular}{llllll}
\hline \hline run & $M_{\mathrm{ej}} / M_{\odot}$ & $R_{0} / R_{\odot}$ & $E_{51}$ & forced $\chi_{\mathrm{abs}}$ & Mixed $\mathrm{H}$ \\
\hline $14 \mathrm{E} 0.7$ & 14.67 & 48.5 & 0.72 & no & yes \\
$14 \mathrm{E} 1$ & 14.67 & 48.5 & 1.03 & no & yes \\
$14 \mathrm{E} 1.21$ & 14.67 & 48.5 & 1.21 & no & yes \\
$14 \mathrm{E} 1.3$ & 14.67 & 48.5 & 1.34 & no & yes \\
$14 \mathrm{E} 1 \mathrm{M}$ & 14.67 & 48.5 & 1.04 & no & yes \\
$14 \mathrm{E} 1 \mathrm{U}$ & 14.67 & 48.5 & 1.01 & no & no \\
14E1.2U & 14.67 & 48.5 & 1.20 & no & no \\
14E1S & 14.67 & 48.5 & 1.03 & no & yes \\
$14 \mathrm{E} 1 \mathrm{~A}$ & 14.67 & 48.5 & 1.01 & yes & yes \\
$14 \mathrm{E} 1.25 \mathrm{H}$ & 14.67 & 48.5 & 1.25 & no & yes \\
14E1.26R & 14.67 & 40 & 1.26 & no & yes \\
14E1.34R & 14.67 & 40 & 1.34 & no & yes \\
14E1.45R & 14.67 & 40 & 1.45 & no & yes \\
14E1.4R6 & 14.67 & 58 & 1.42 & no & yes \\
\hline
\end{tabular}

Table 2: Predictions for the first maximum light

\begin{tabular}{lclllll}
\hline \hline run & $t$, day & $L_{\text {bol }}$, ergs s$^{-1}$ & $T_{\mathrm{c}}, \mathrm{K}$ & $T_{\text {eff }}, \mathrm{K}$ & $R_{\tau=2 / 3}, \mathrm{~cm}$ & $\int_{0}^{2=\mathrm{d}} L d t$, ergs \\
\hline $14 \mathrm{E} 0.7$ & .08960 & $4.217 \times 10^{44}$ & $1.074 \times 10^{6}$ & $4.71 \times 10^{5}$ & $3.32 \times 10^{12}$ & $1.07 \times 10^{47}$ \\
$14 \mathrm{E} 1$ & .07637 & $6.751 \times 10^{44}$ & $1.219 \times 10^{6}$ & $5.28 \times 10^{5}$ & $3.32 \times 10^{12}$ & $1.40 \times 10^{47}$ \\
$14 \mathrm{E} 1.3$ & .06726 & $9.466 \times 10^{44}$ & $1.339 \times 10^{6}$ & $5.73 \times 10^{5}$ & $3.32 \times 10^{12}$ & $1.77 \times 10^{47}$ \\
$14 \mathrm{E} 1 \mathrm{U}$ & .07692 & $6.616 \times 10^{44}$ & $1.207 \times 10^{6}$ & $5.24 \times 10^{5}$ & $3.32 \times 10^{12}$ & $1.41 \times 10^{47}$ \\
$14 \mathrm{E} 1.2 \mathrm{U}$ & .07161 & $7.939 \times 10^{44}$ & $1.268 \times 10^{6}$ & $5.49 \times 10^{5}$ & $3.32 \times 10^{12}$ & $1.58 \times 10^{47}$ \\
$14 \mathrm{E} 1.26 \mathrm{R}$ & .05768 & $8.665 \times 10^{44}$ & $1.428 \times 10^{6}$ & $6.18 \times 10^{5}$ & $2.74 \times 10^{12}$ & $1.30 \times 10^{47}$ \\
$14 \mathrm{E} 1.34 \mathrm{R}$ & .05620 & $9.175 \times 10^{44}$ & $1.451 \times 10^{6}$ & $6.26 \times 10^{5}$ & $2.74 \times 10^{12}$ & $1.36 \times 10^{47}$ \\
$14 \mathrm{E} 1.45 \mathrm{R}$ & .05389 & $1.012 \times 10^{45}$ & $1.497 \times 10^{6}$ & $6.42 \times 10^{5}$ & $2.74 \times 10^{12}$ & $1.44 \times 10^{47}$ \\
$14 \mathrm{E} 1.4 \mathrm{R} 6$ & .07520 & $1.013 \times 10^{45}$ & $1.267 \times 10^{6}$ & $5.30 \times 10^{5}$ & $3.95 \times 10^{12}$ & $2.38 \times 10^{47}$ \\
\hline
\end{tabular}

Note. Maximum $T_{\text {eff }}$ almost coincides with the peak $L_{\mathrm{bol}}$, while maximum $T_{\mathrm{c}}$ is $\sim 100 \mathrm{~s}$ earlier. 


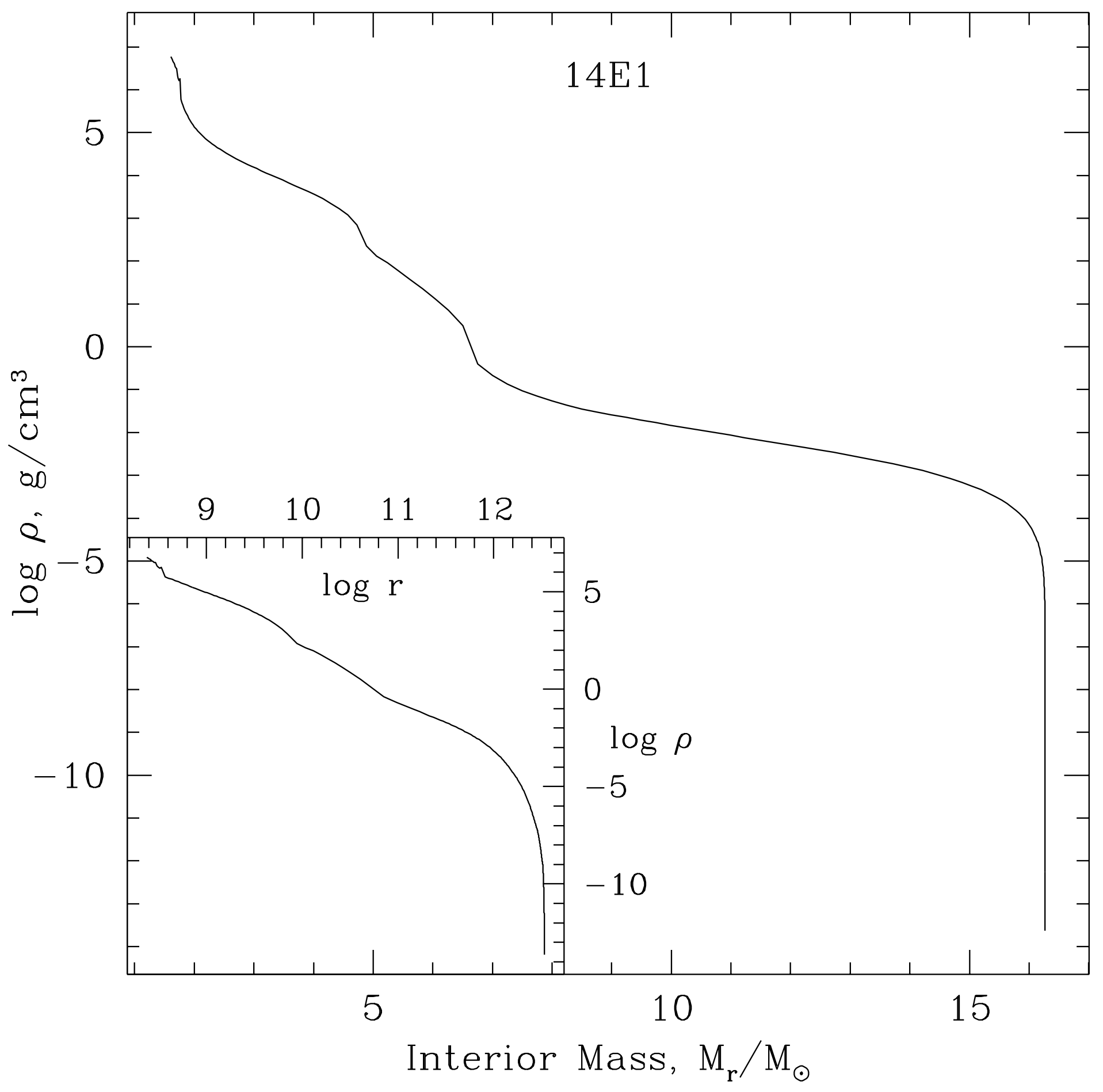




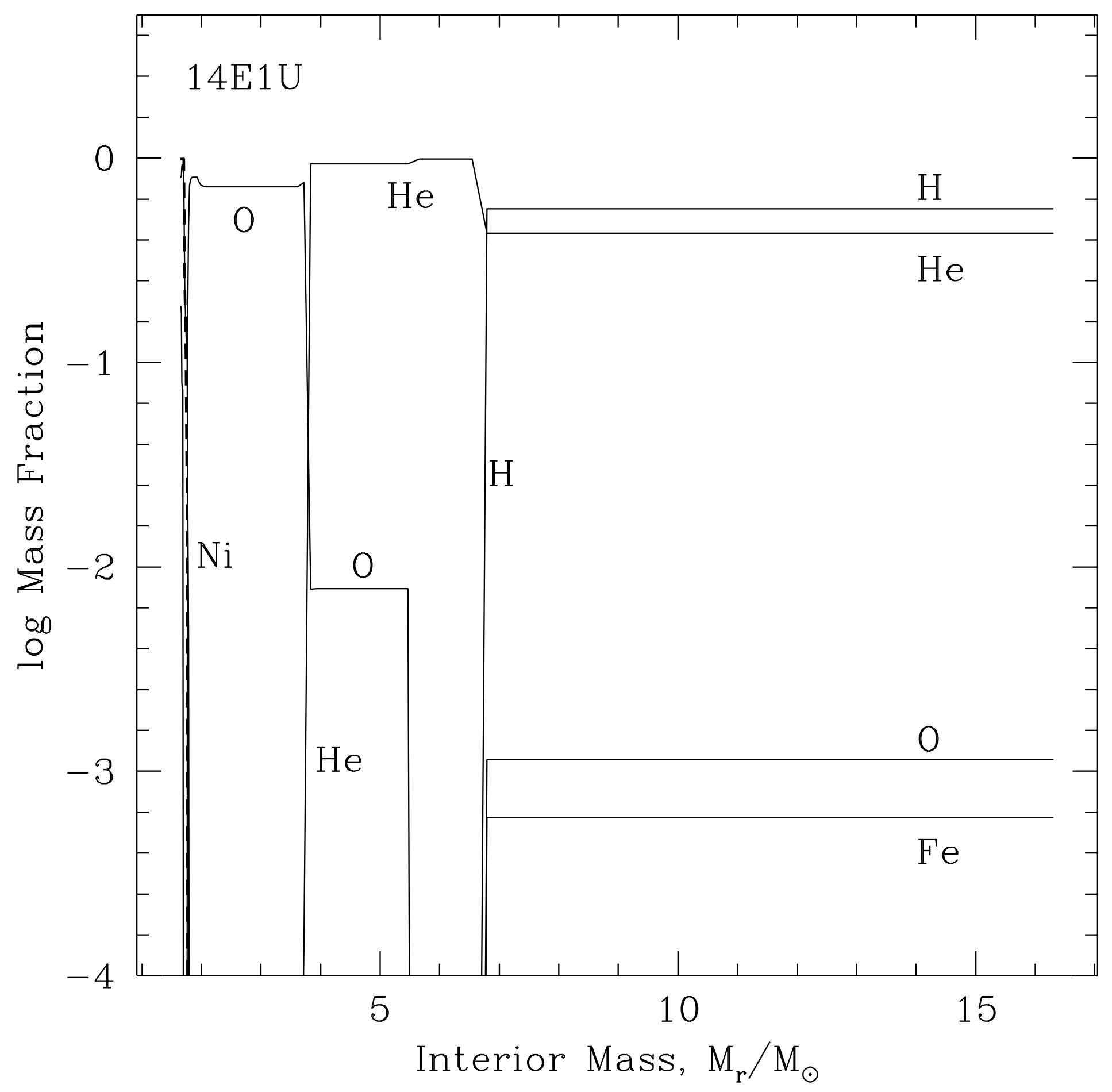




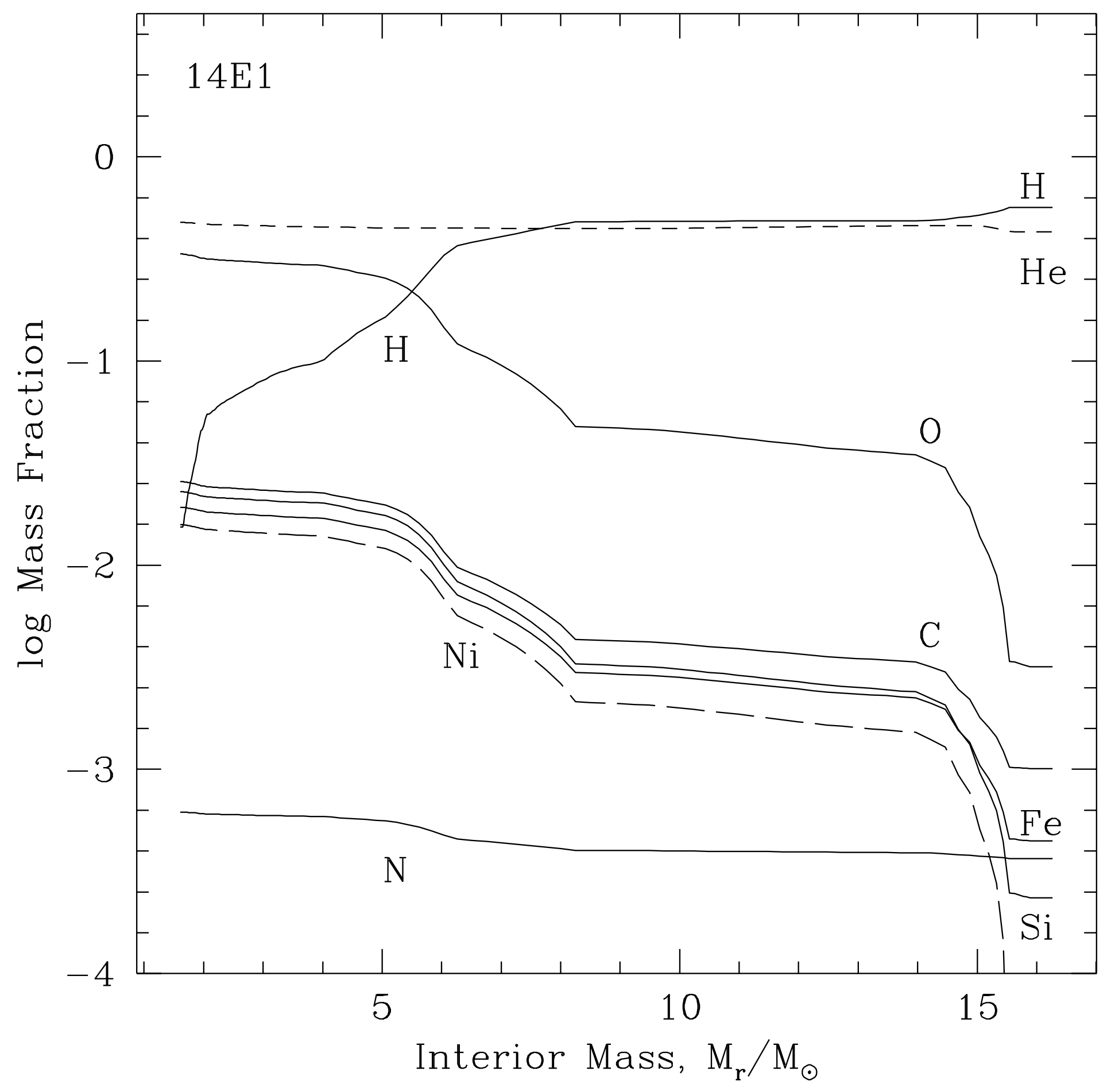




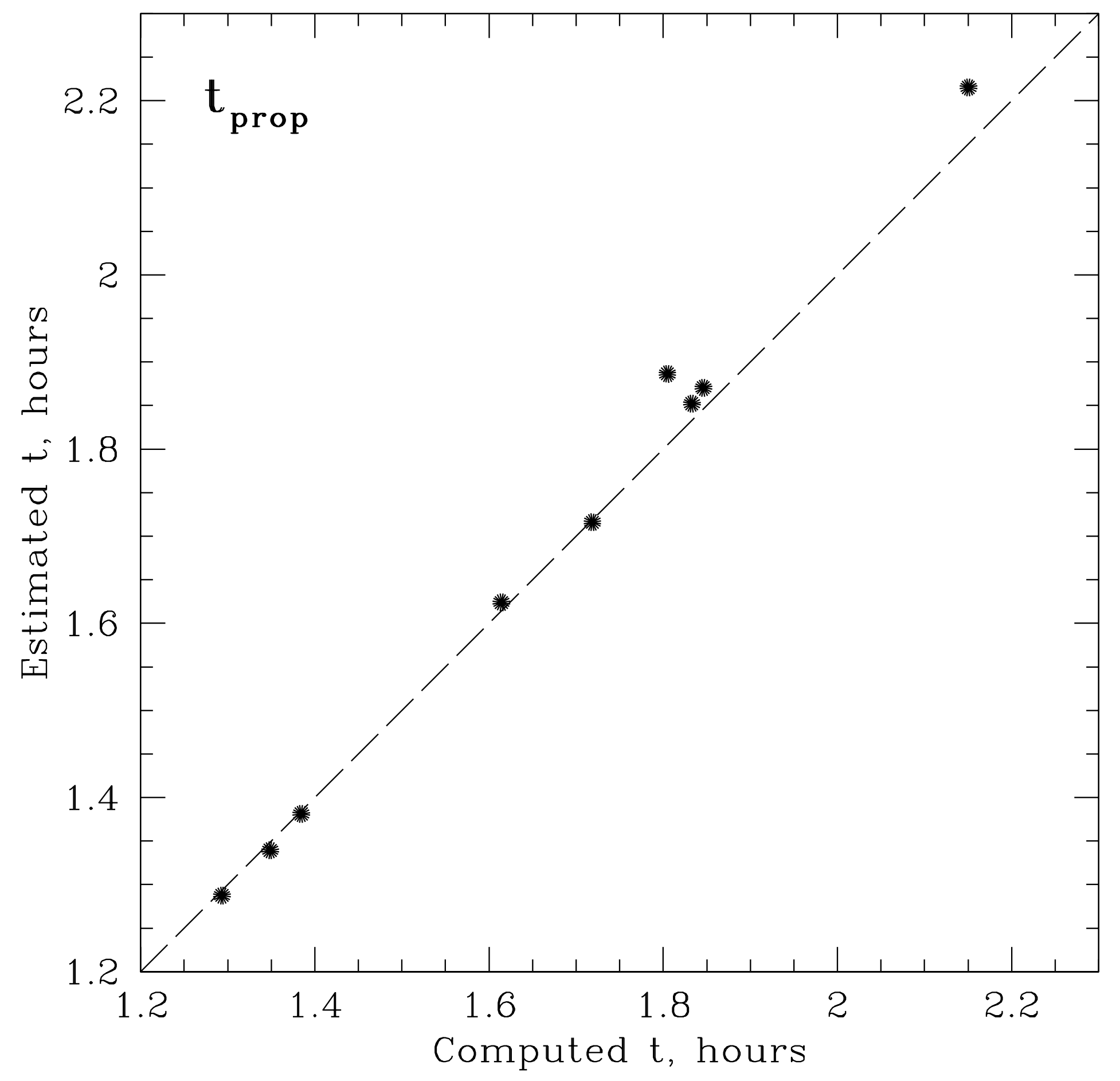




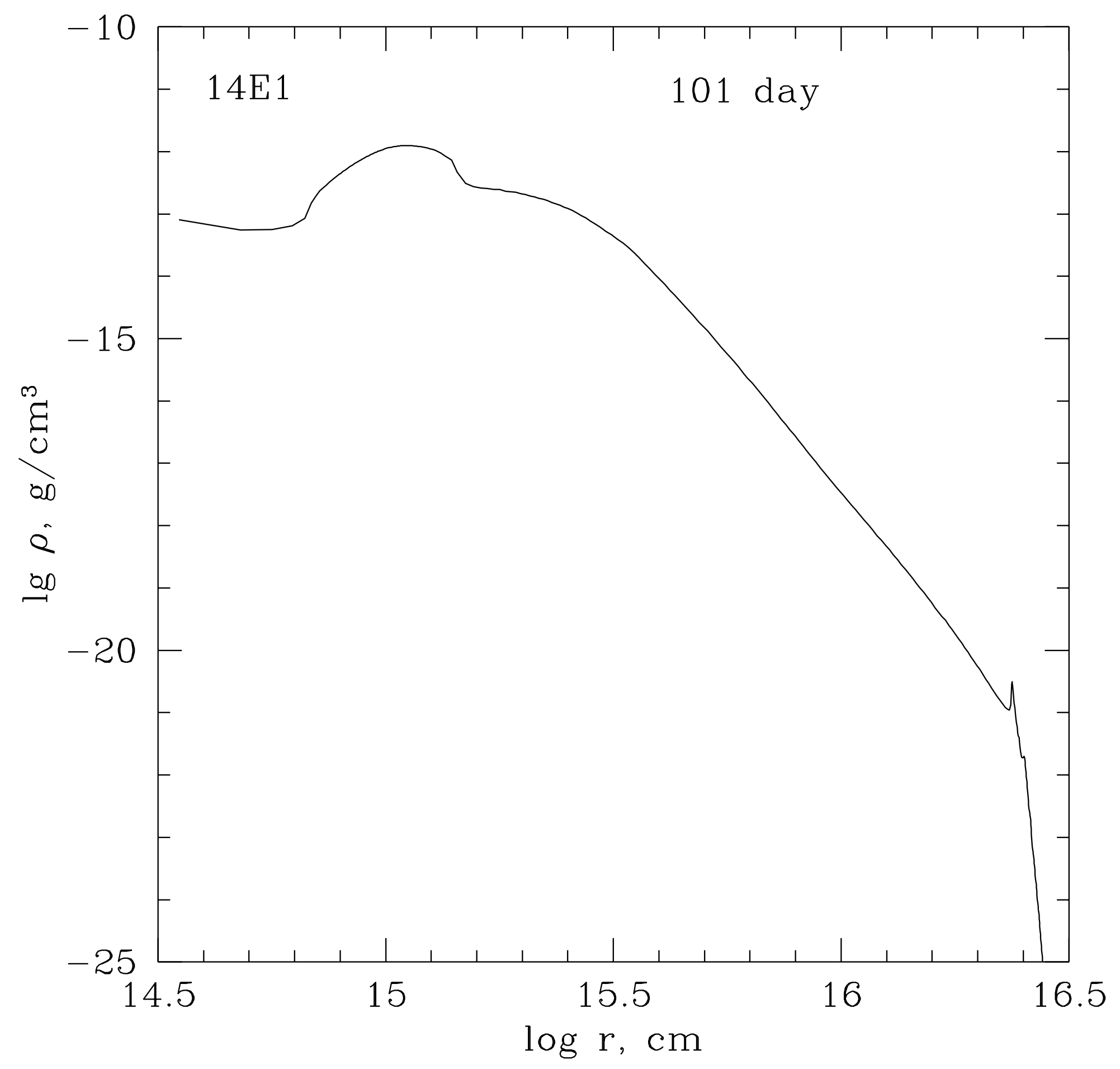




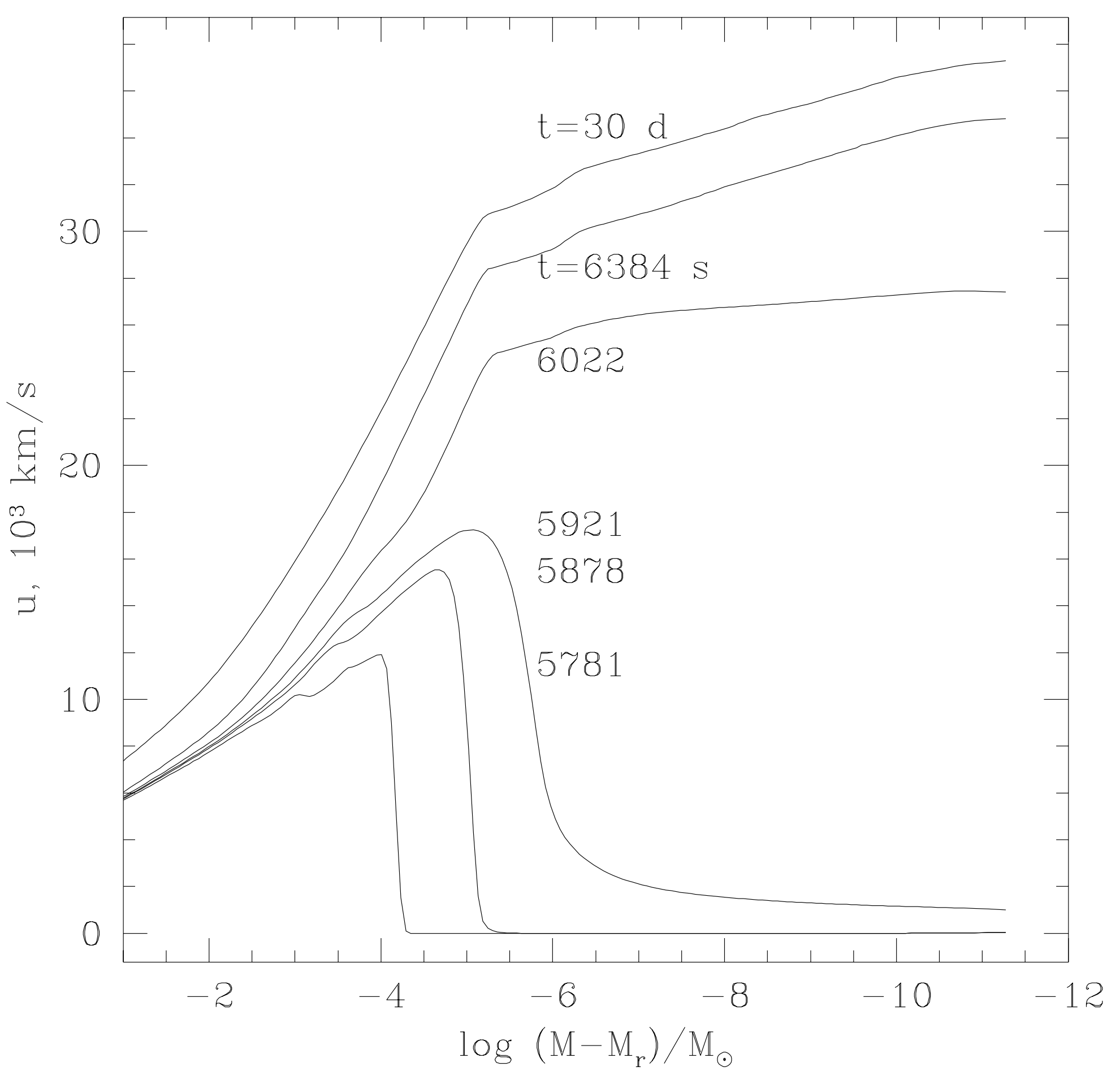




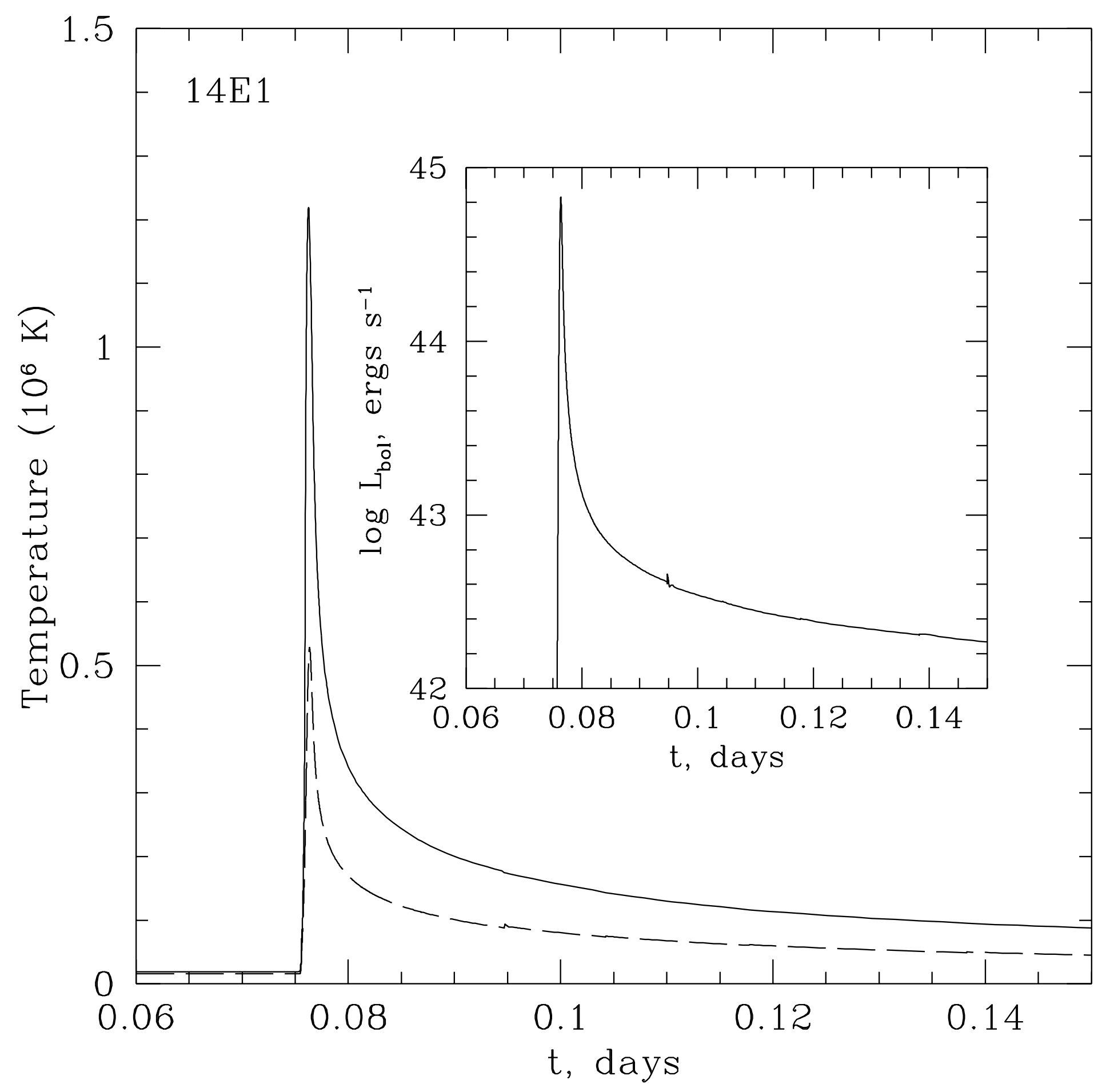




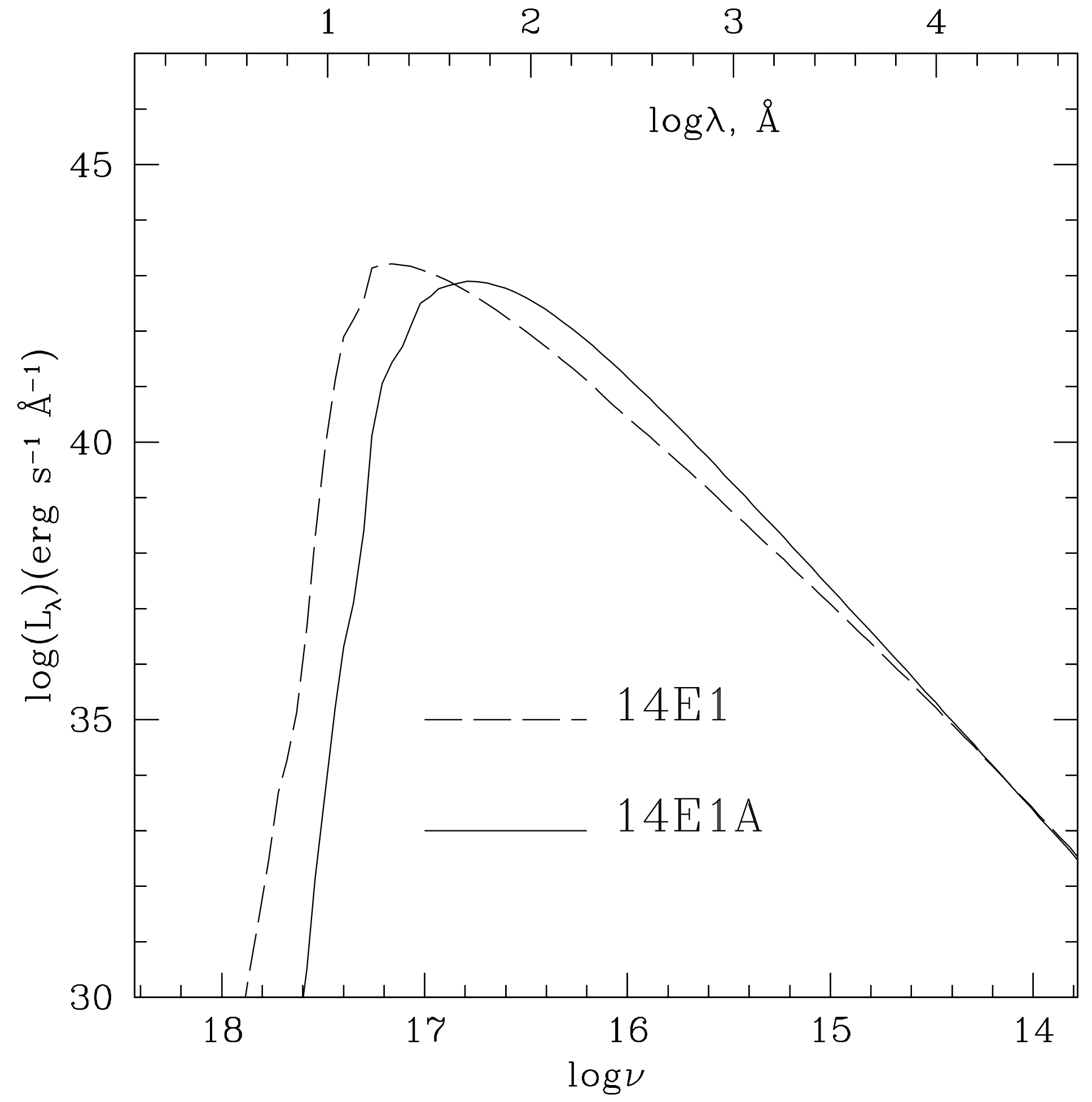




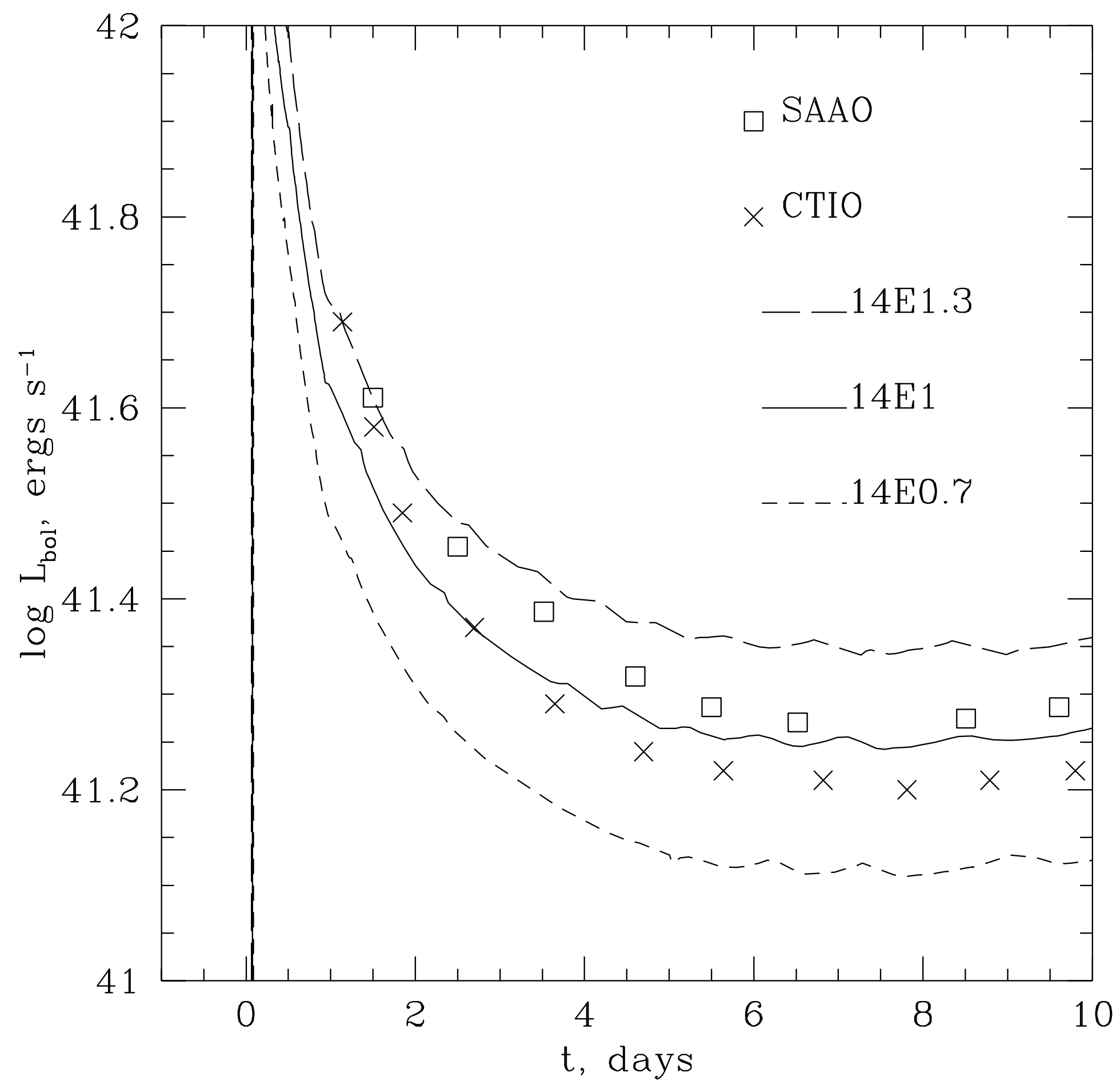




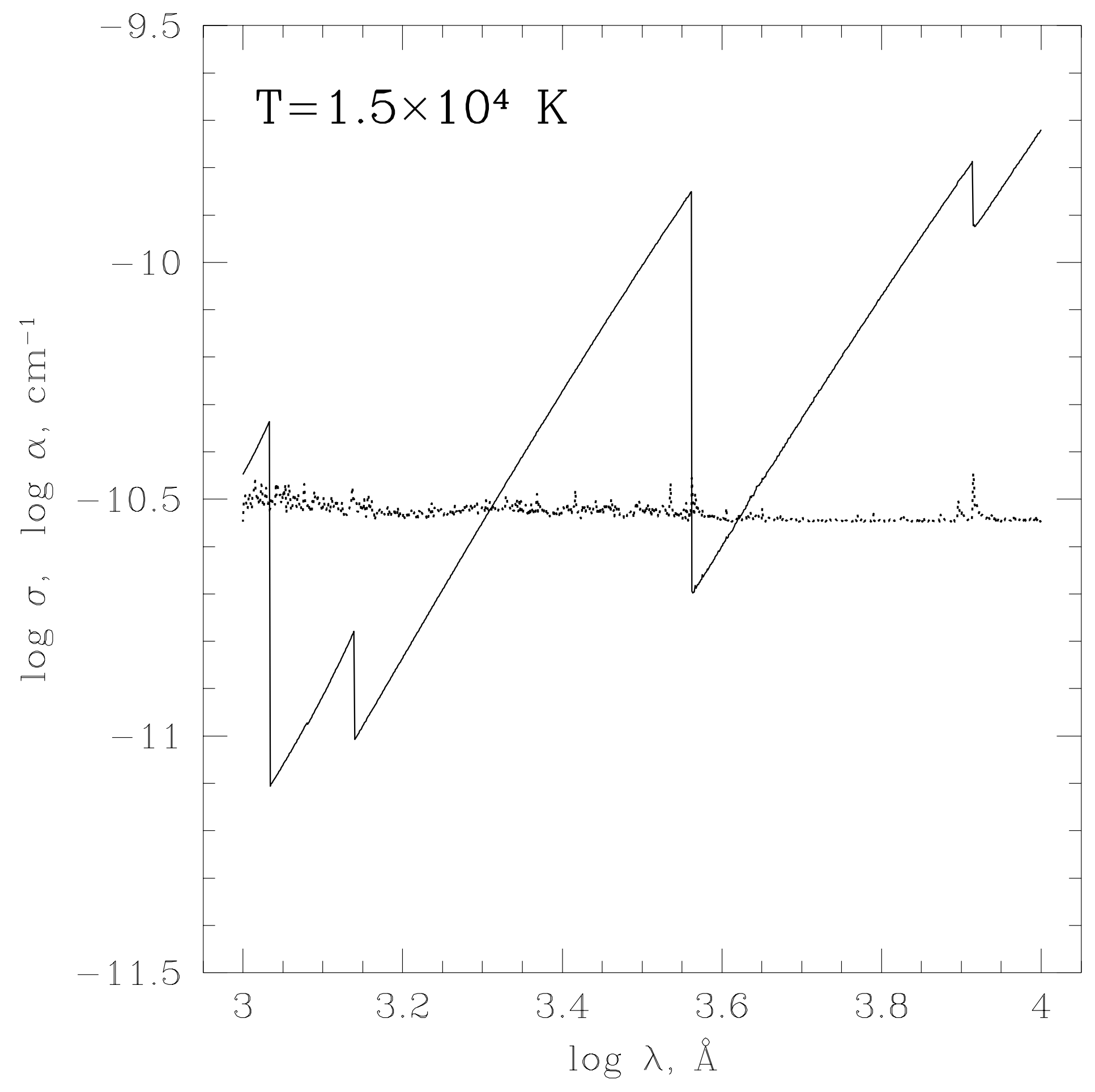




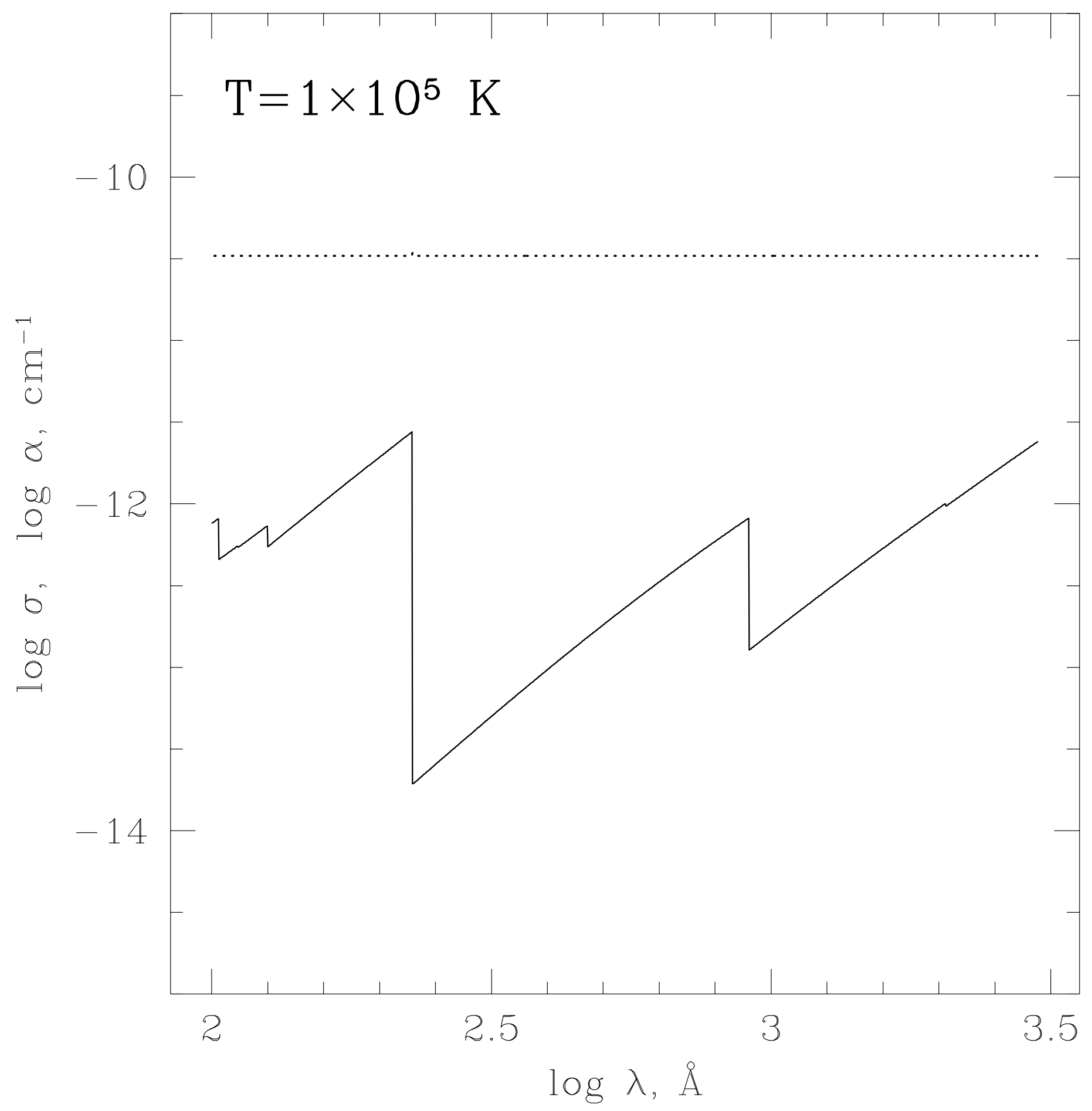




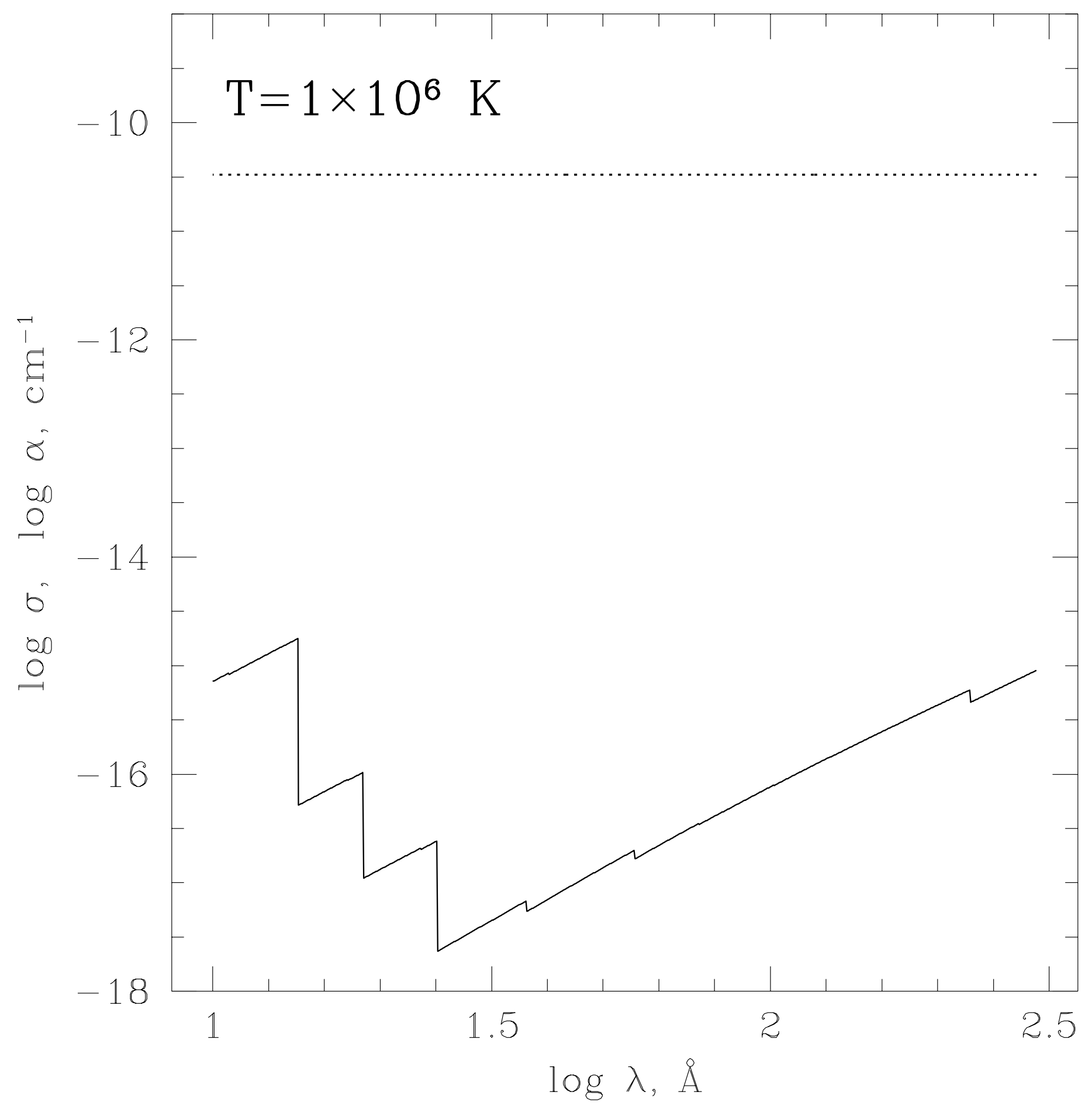




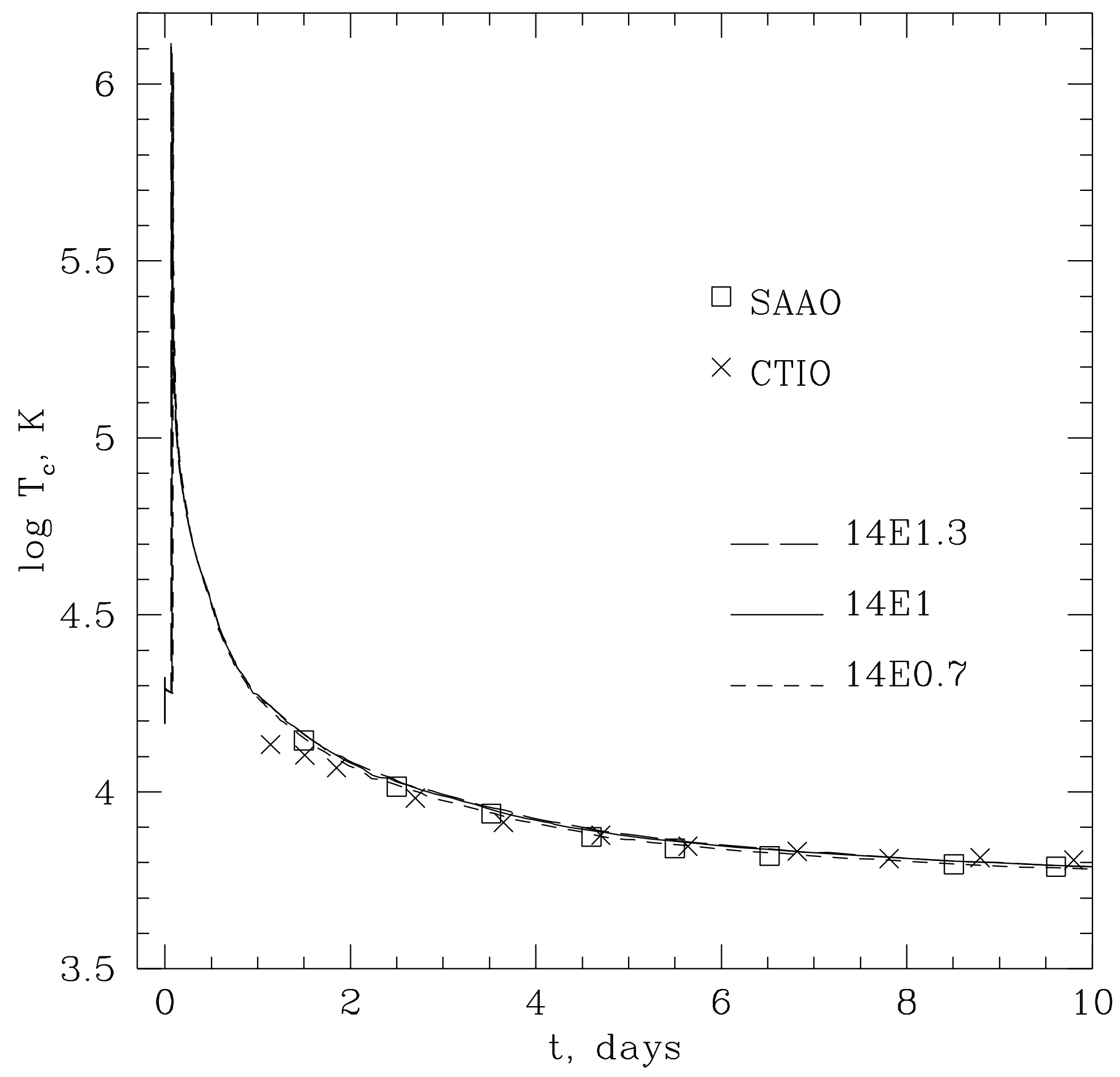




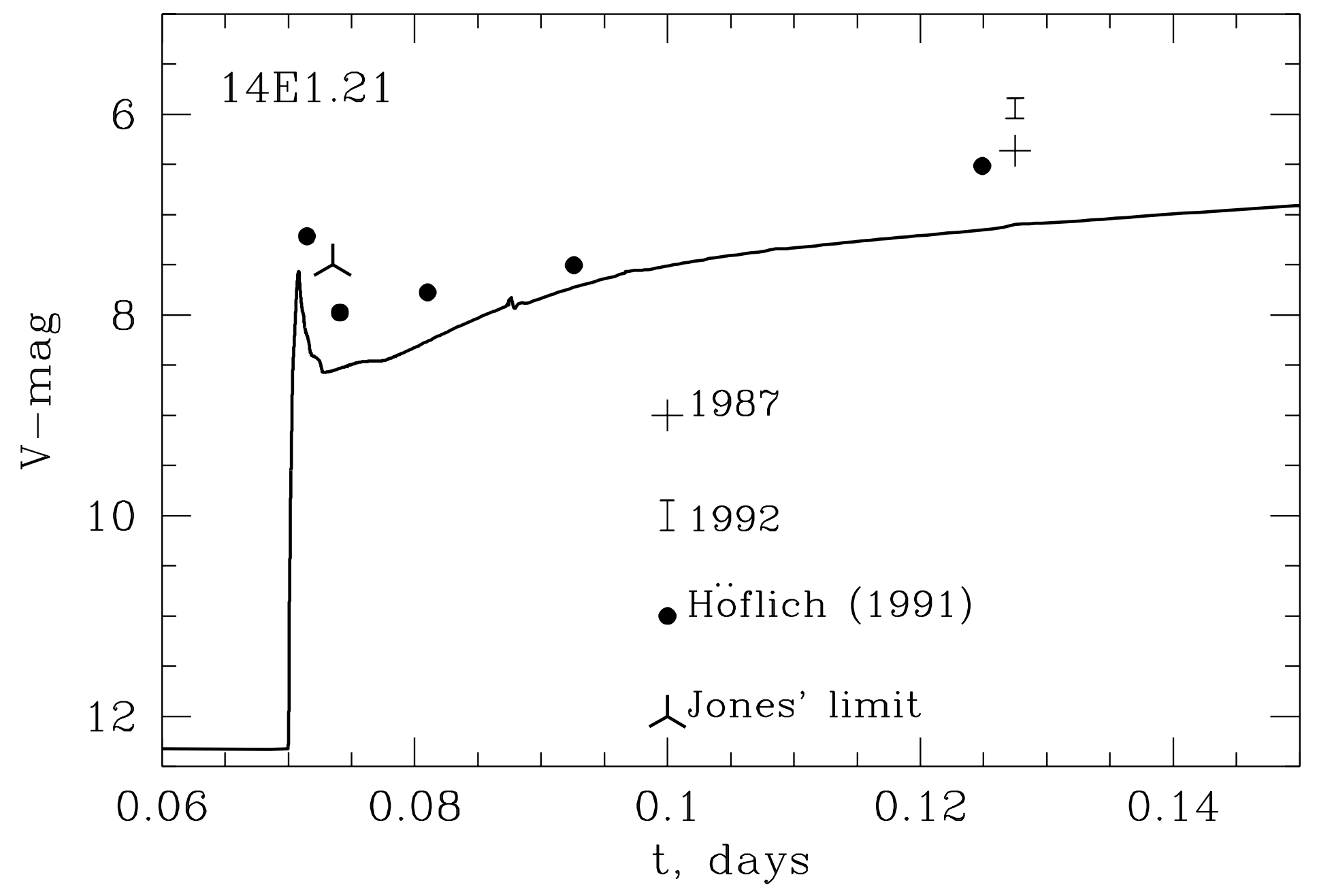




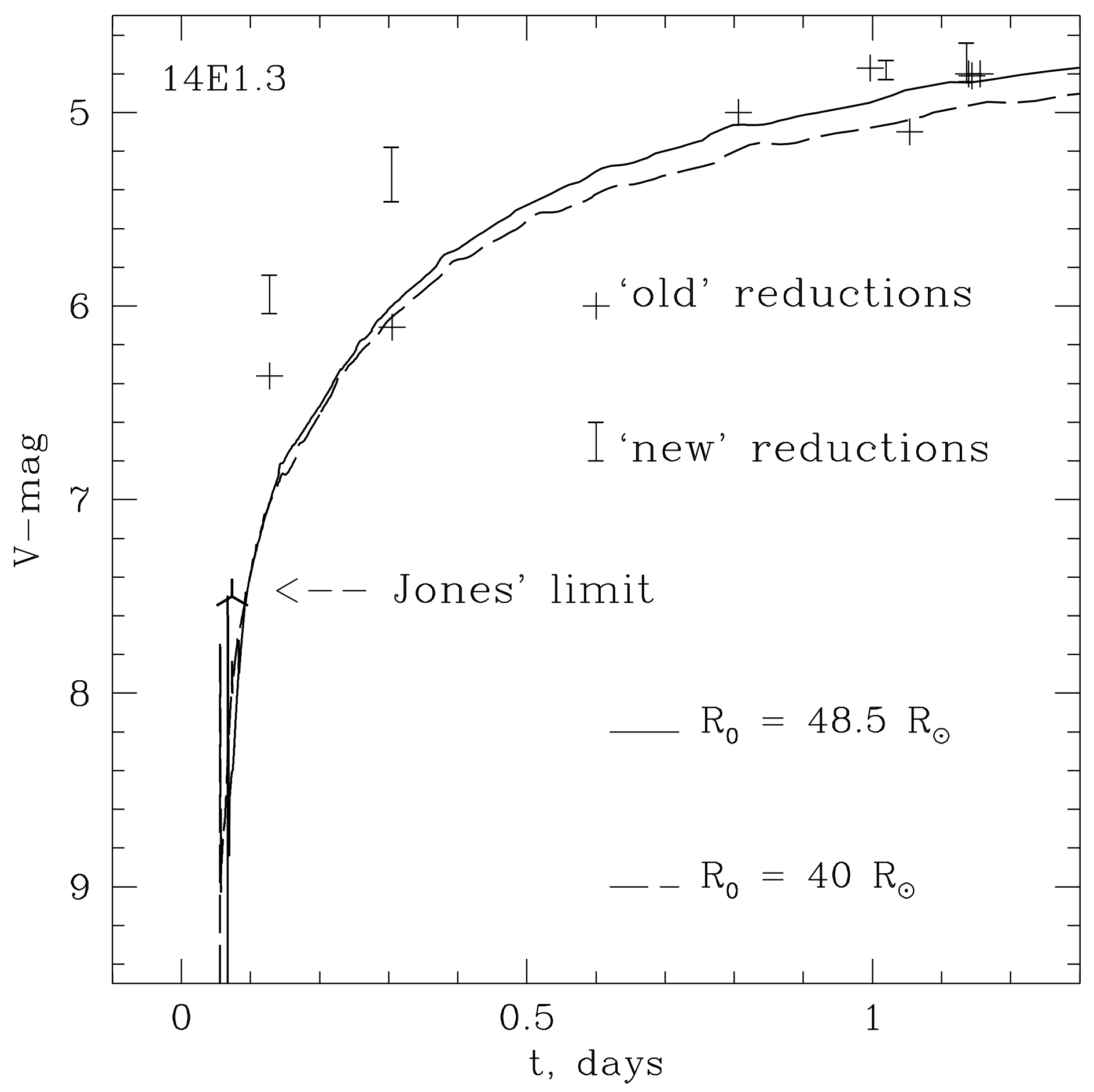




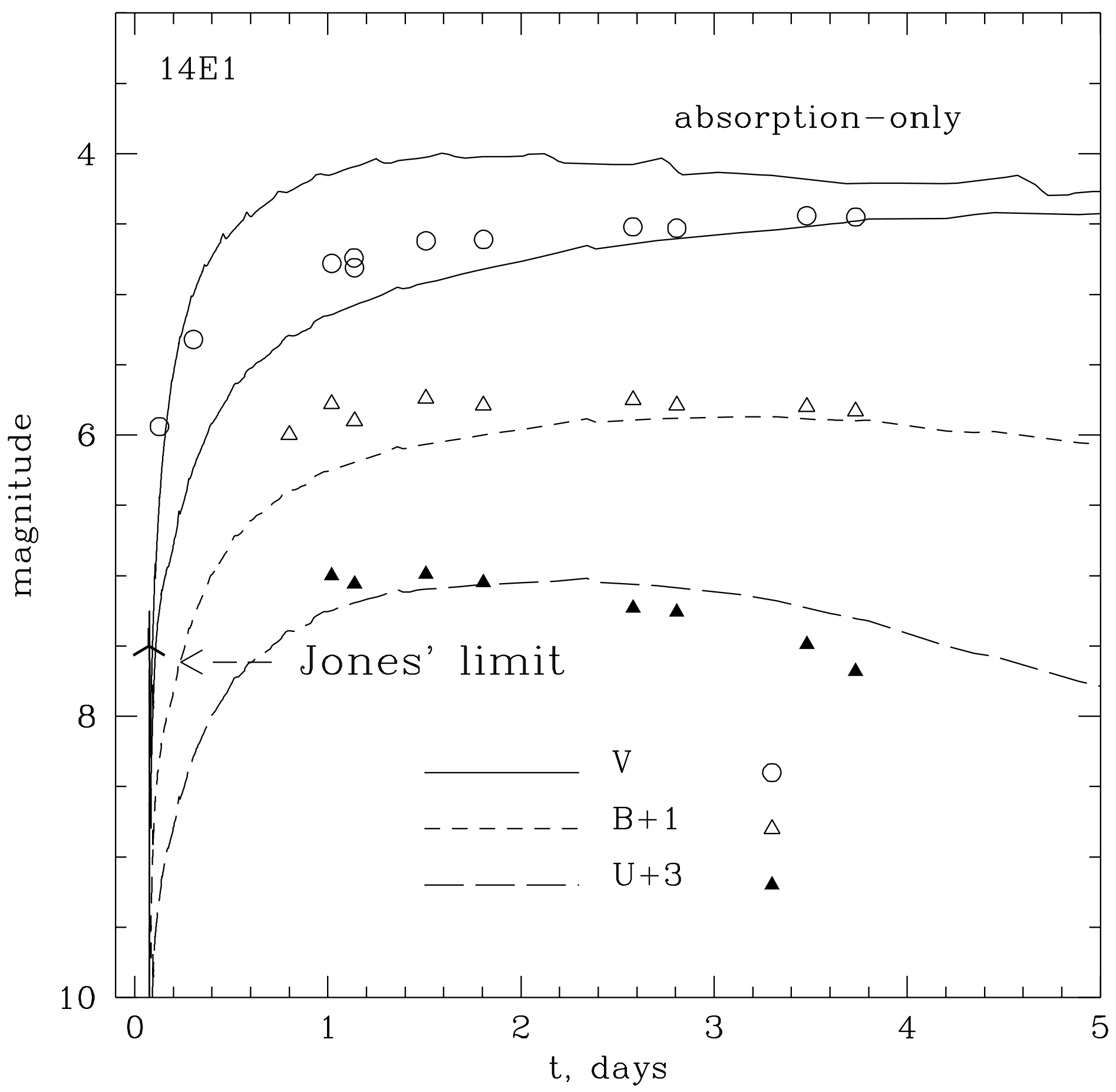




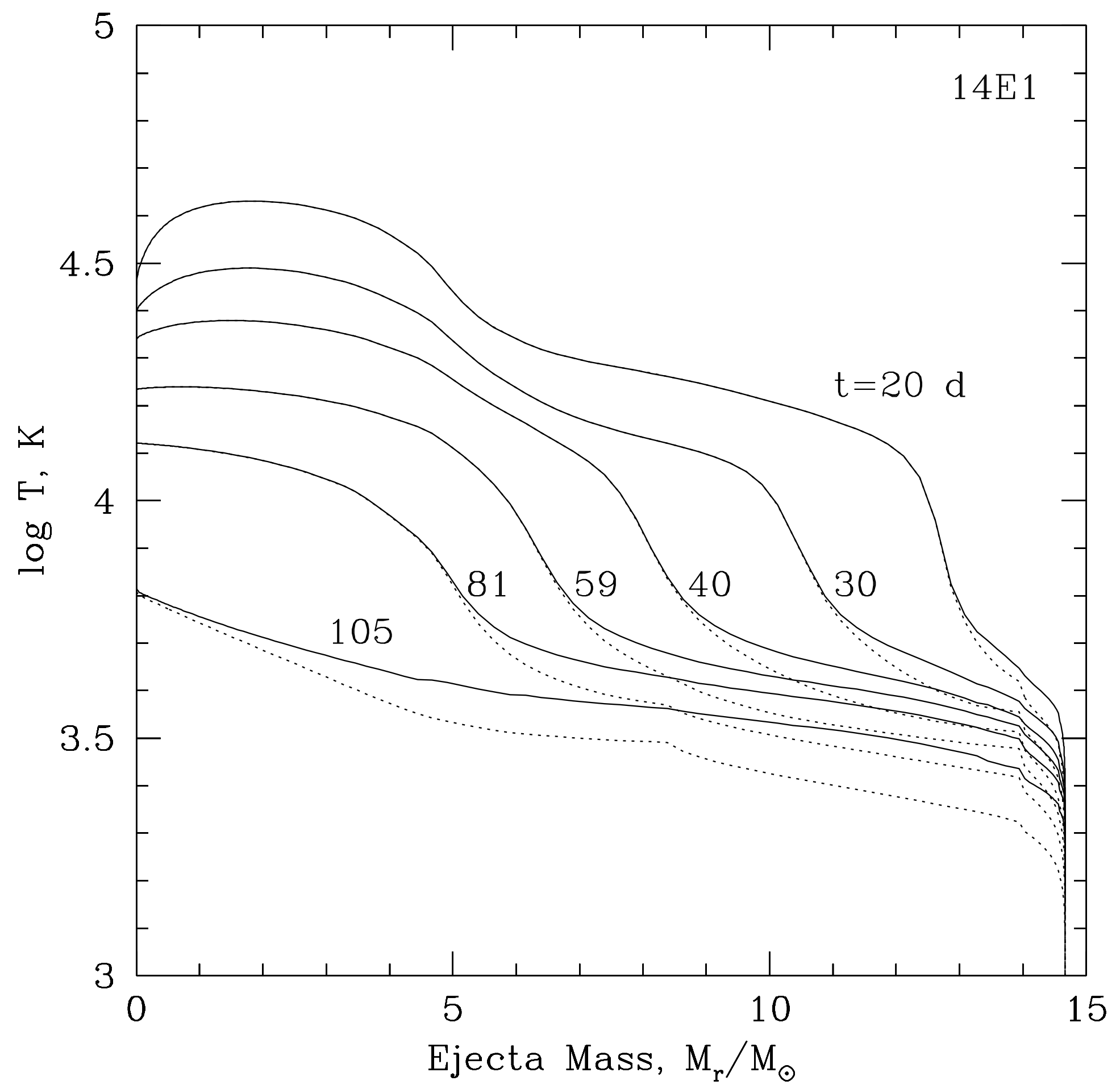




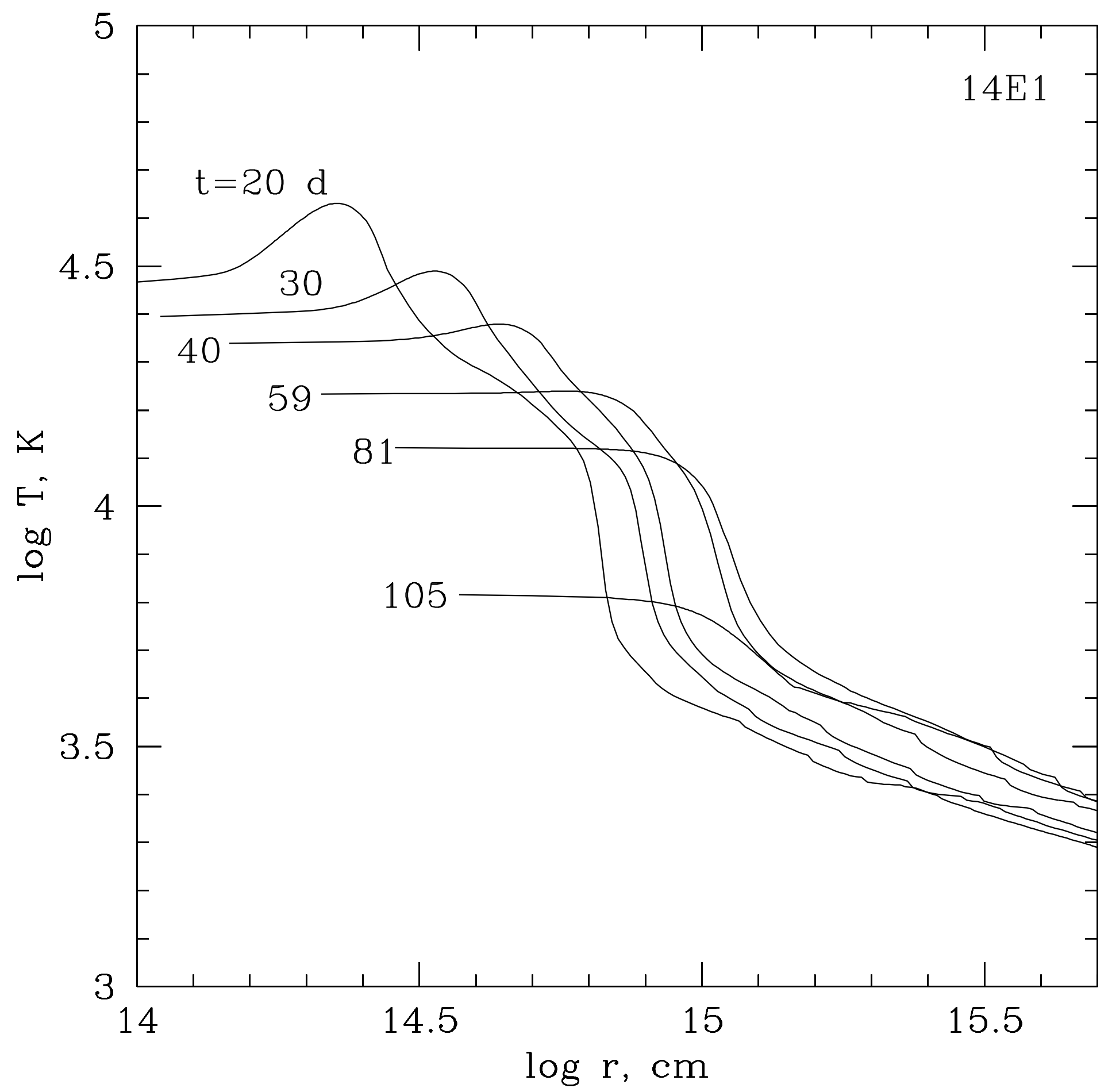




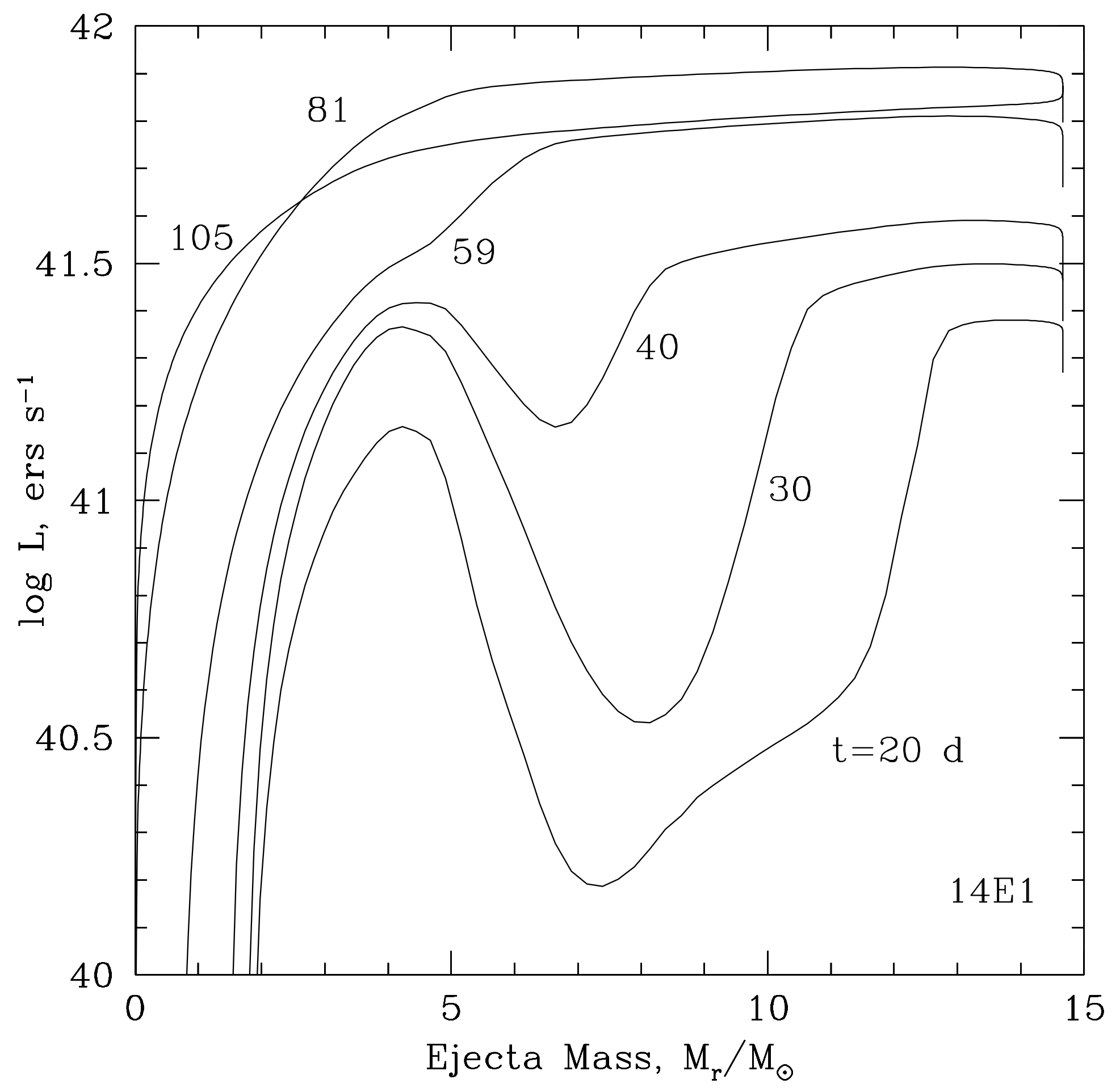




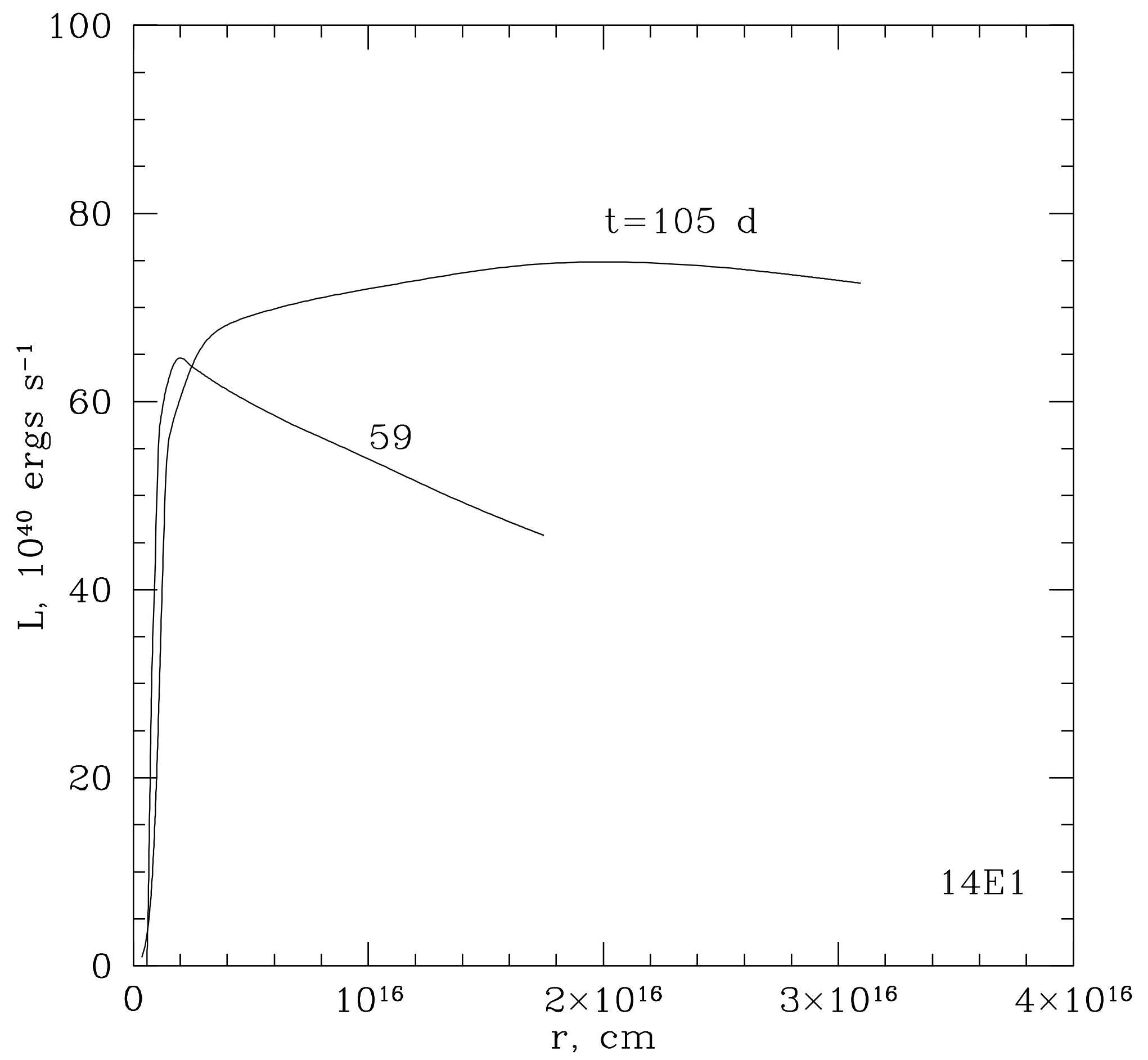




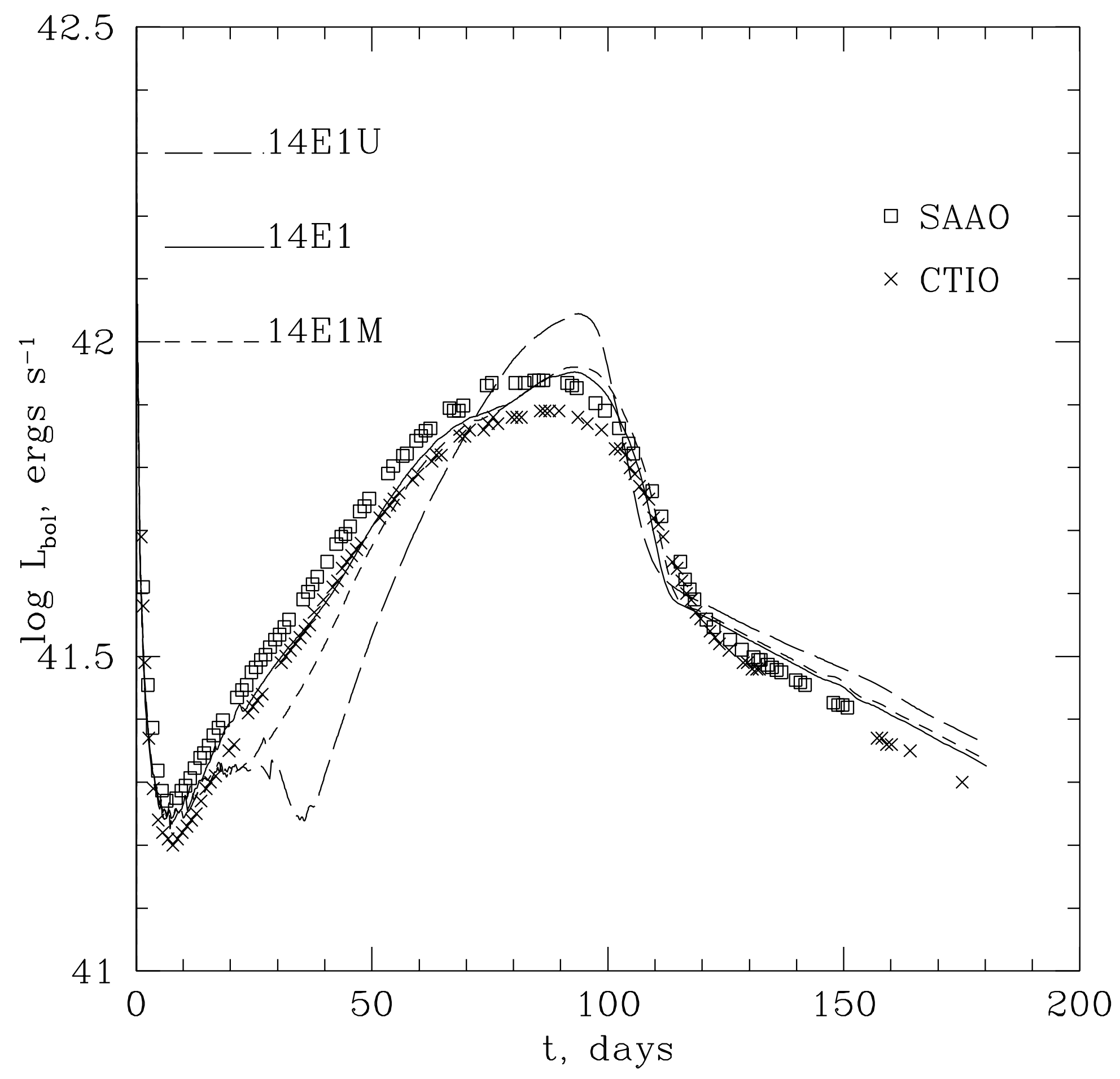




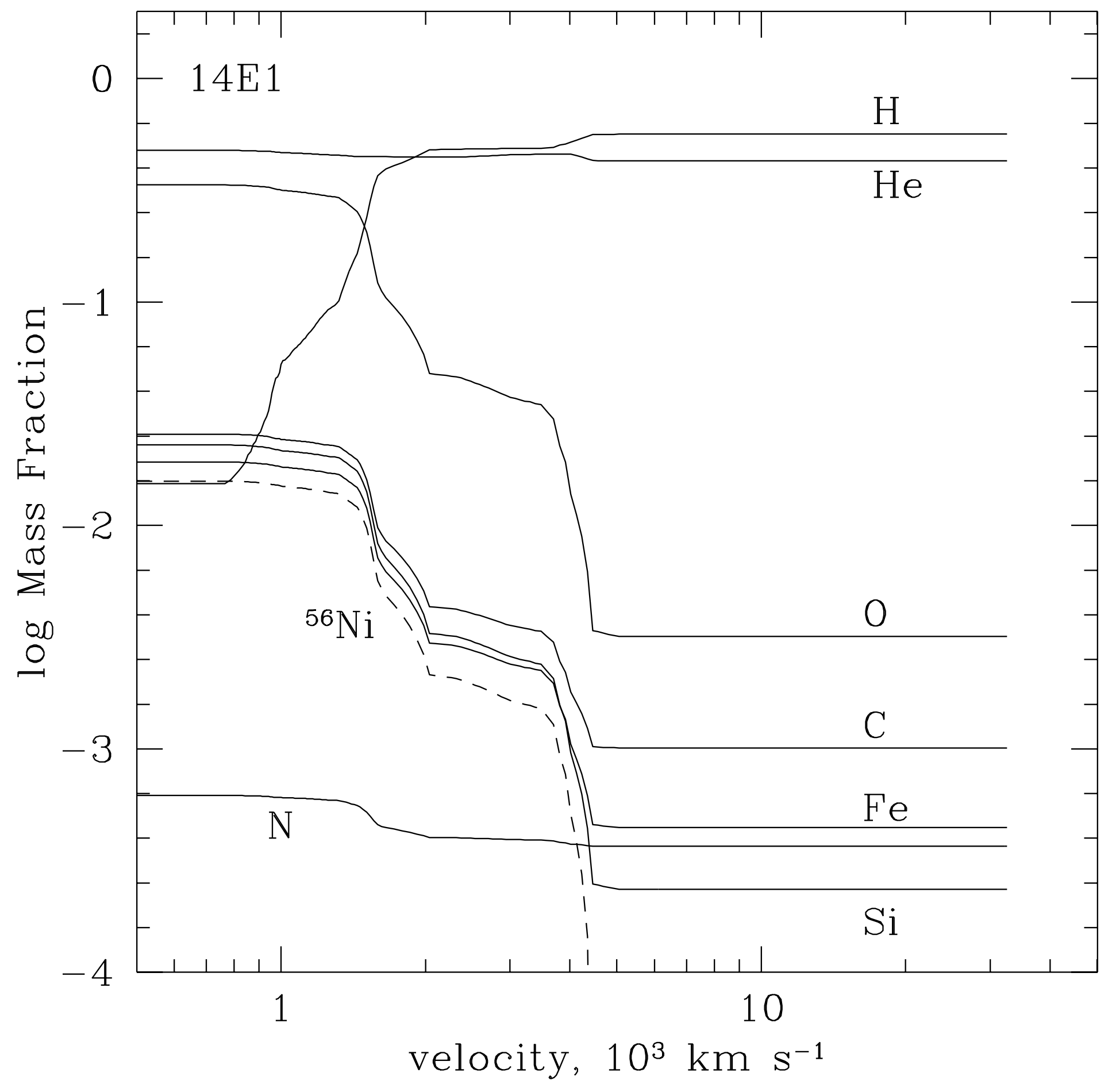




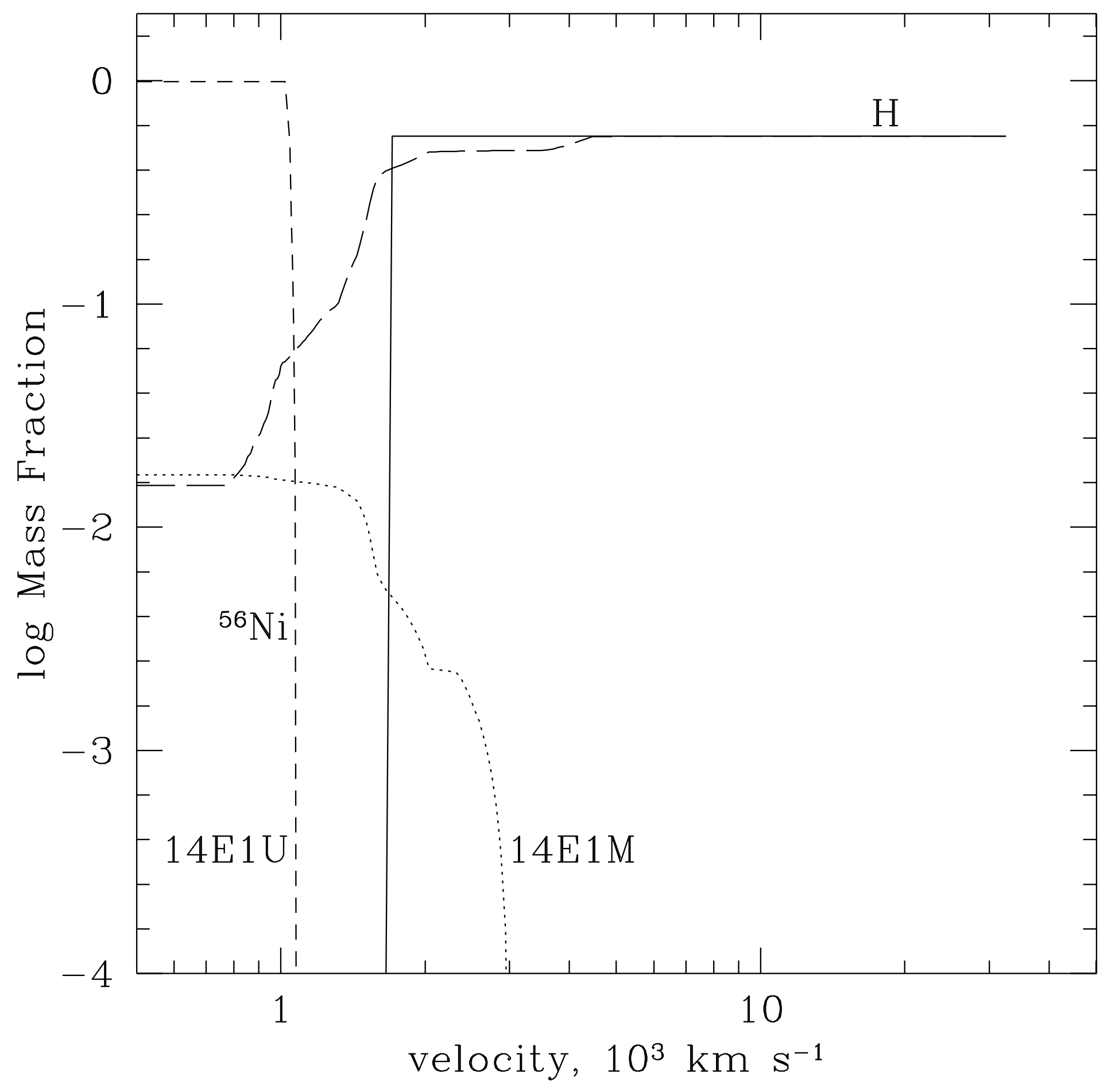




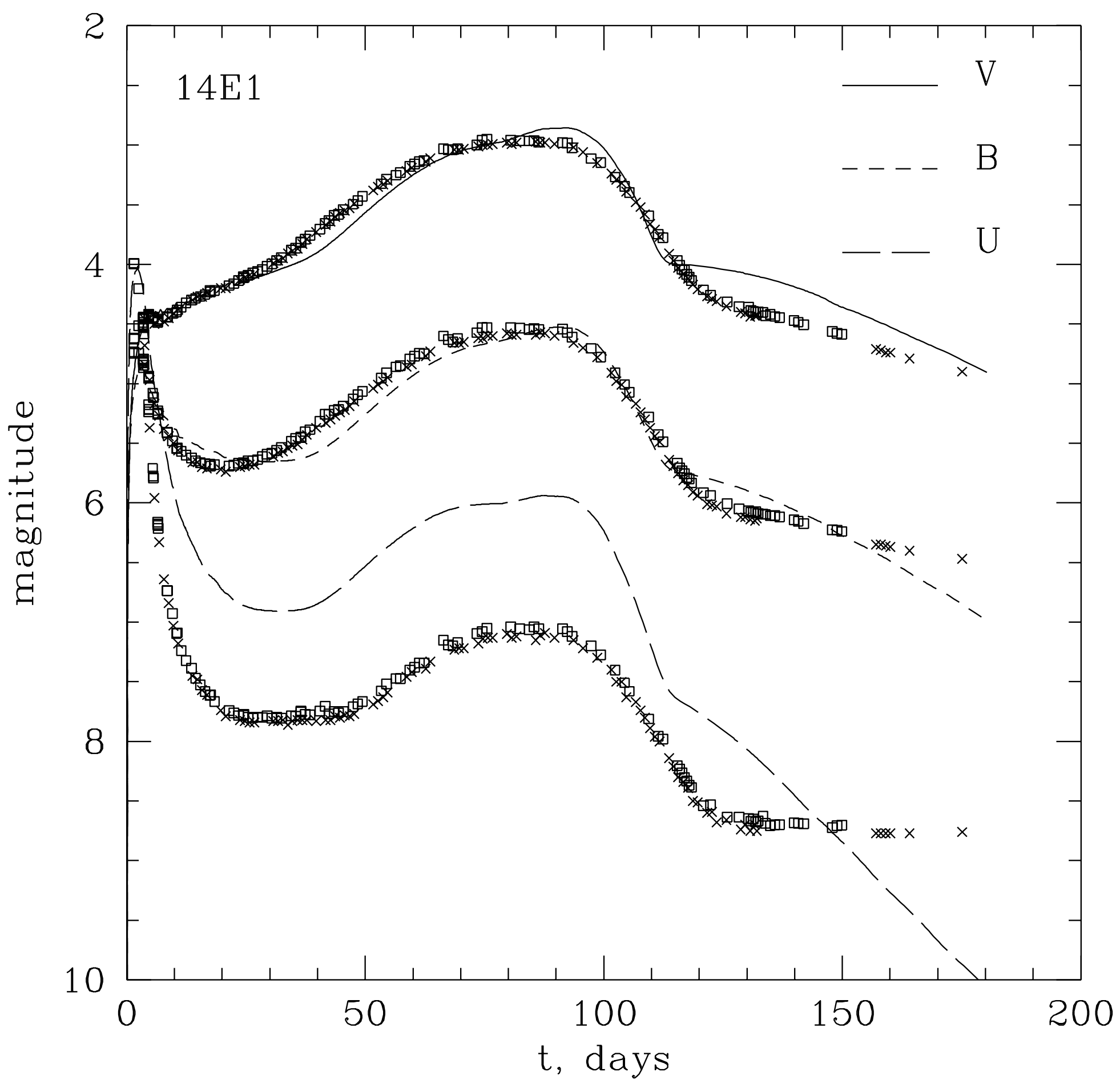




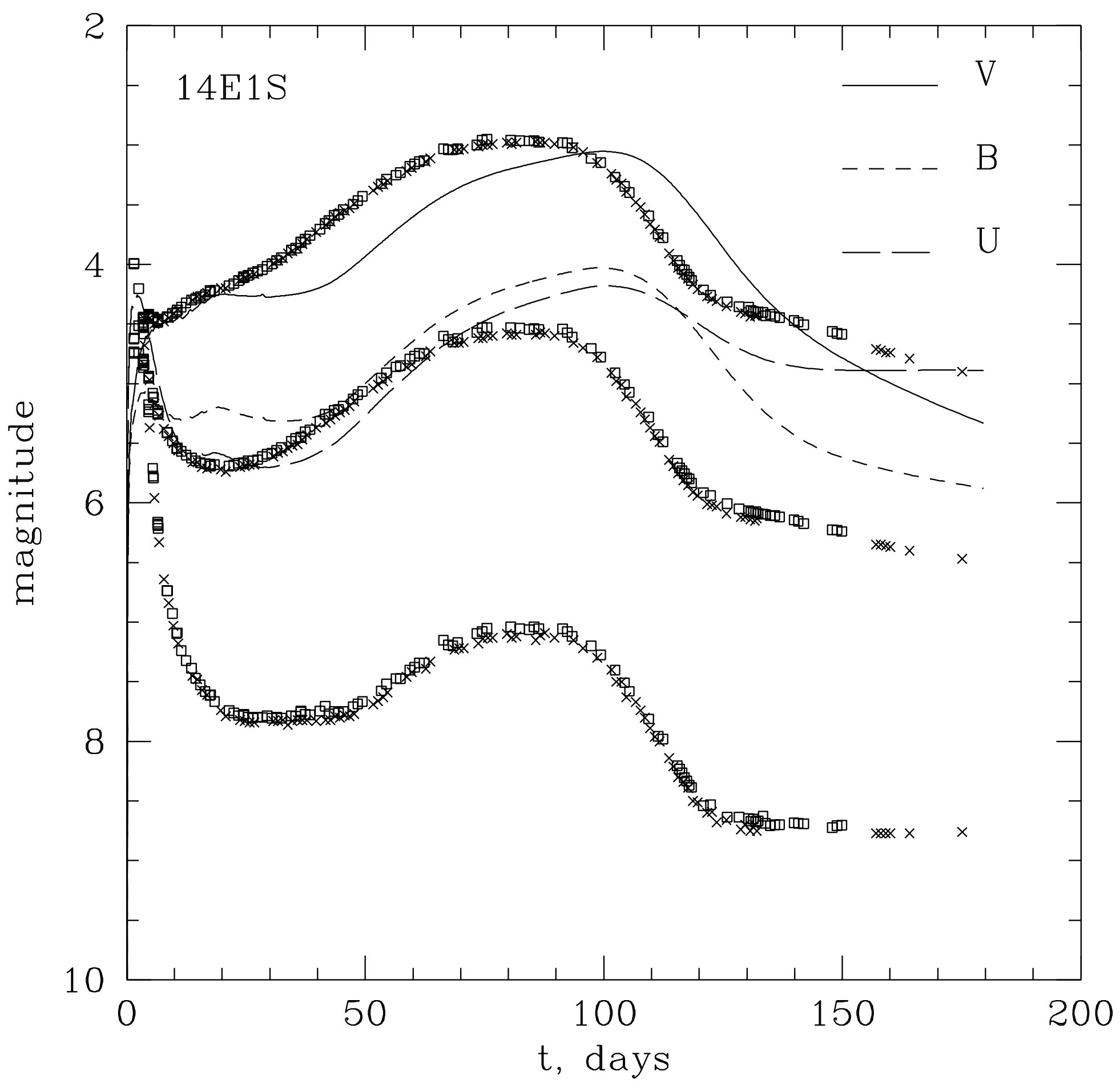



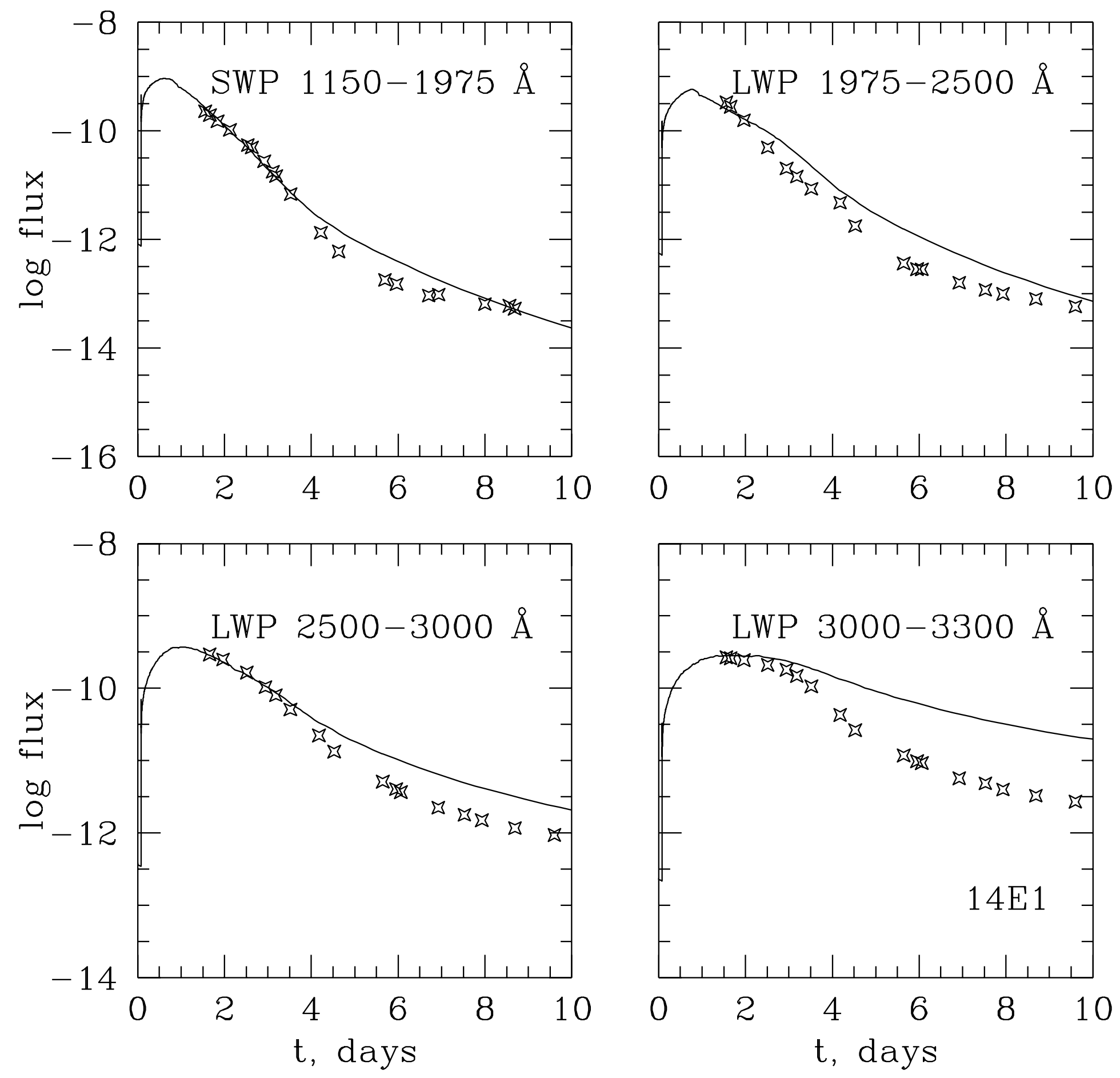

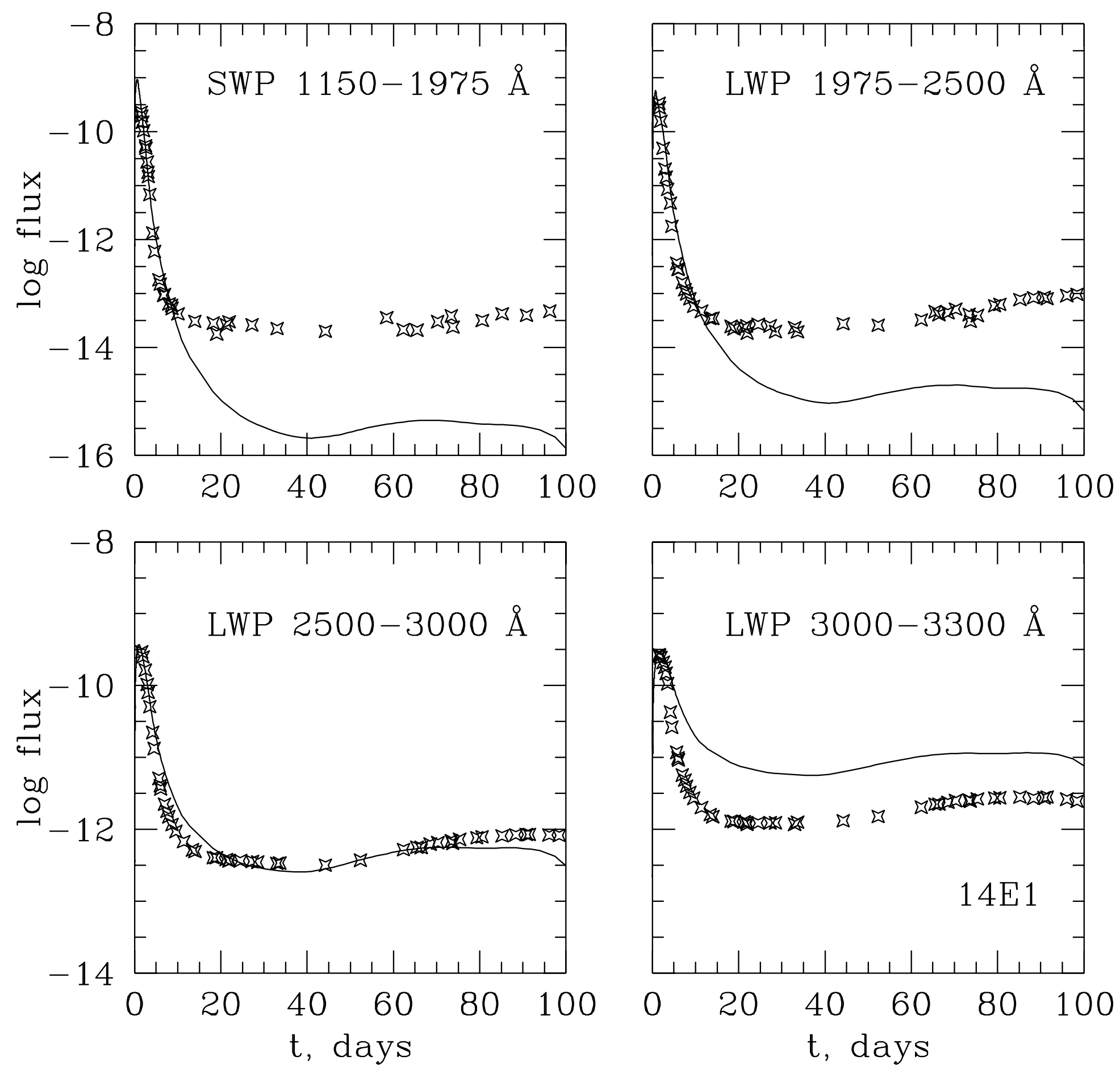


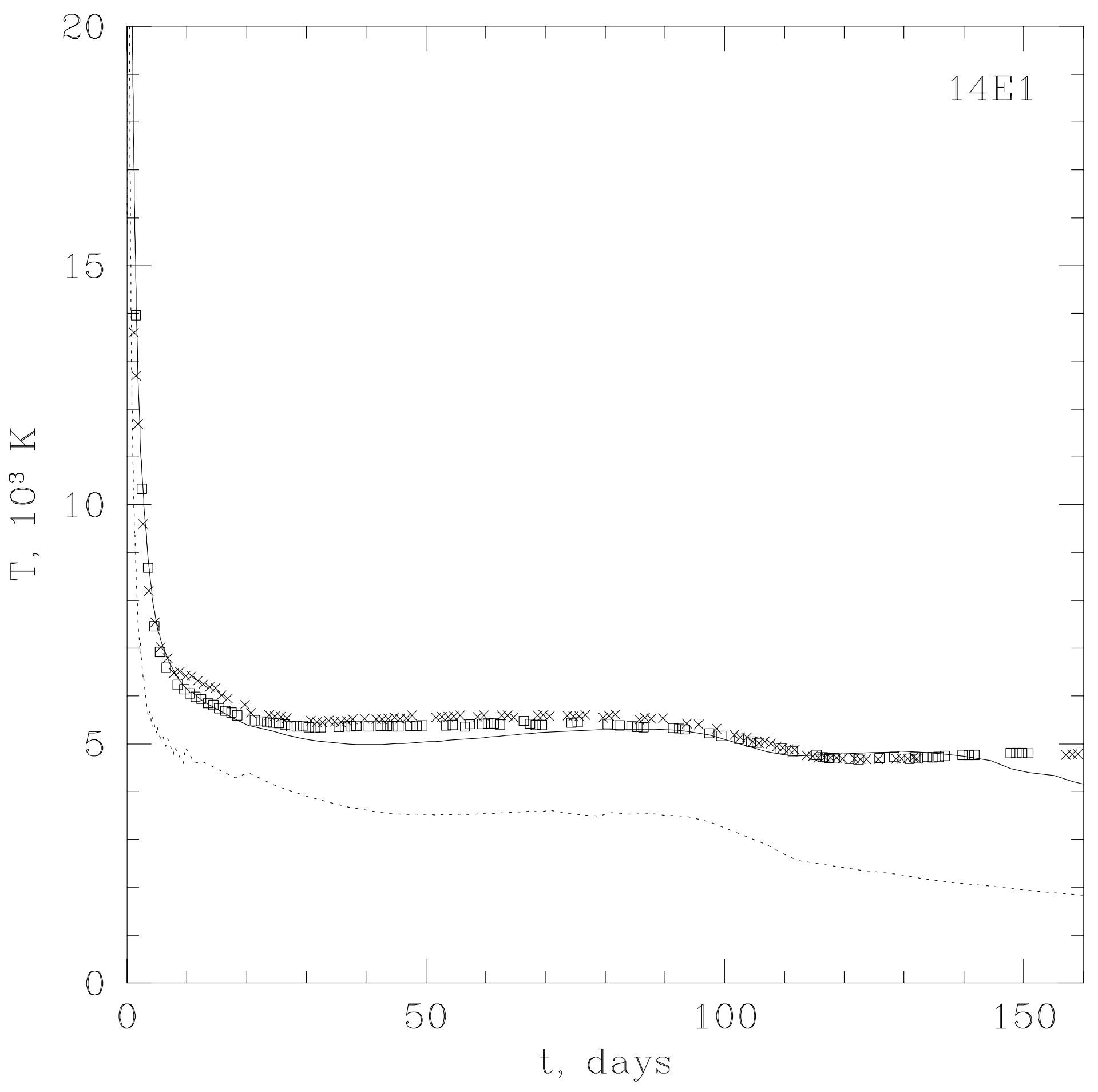




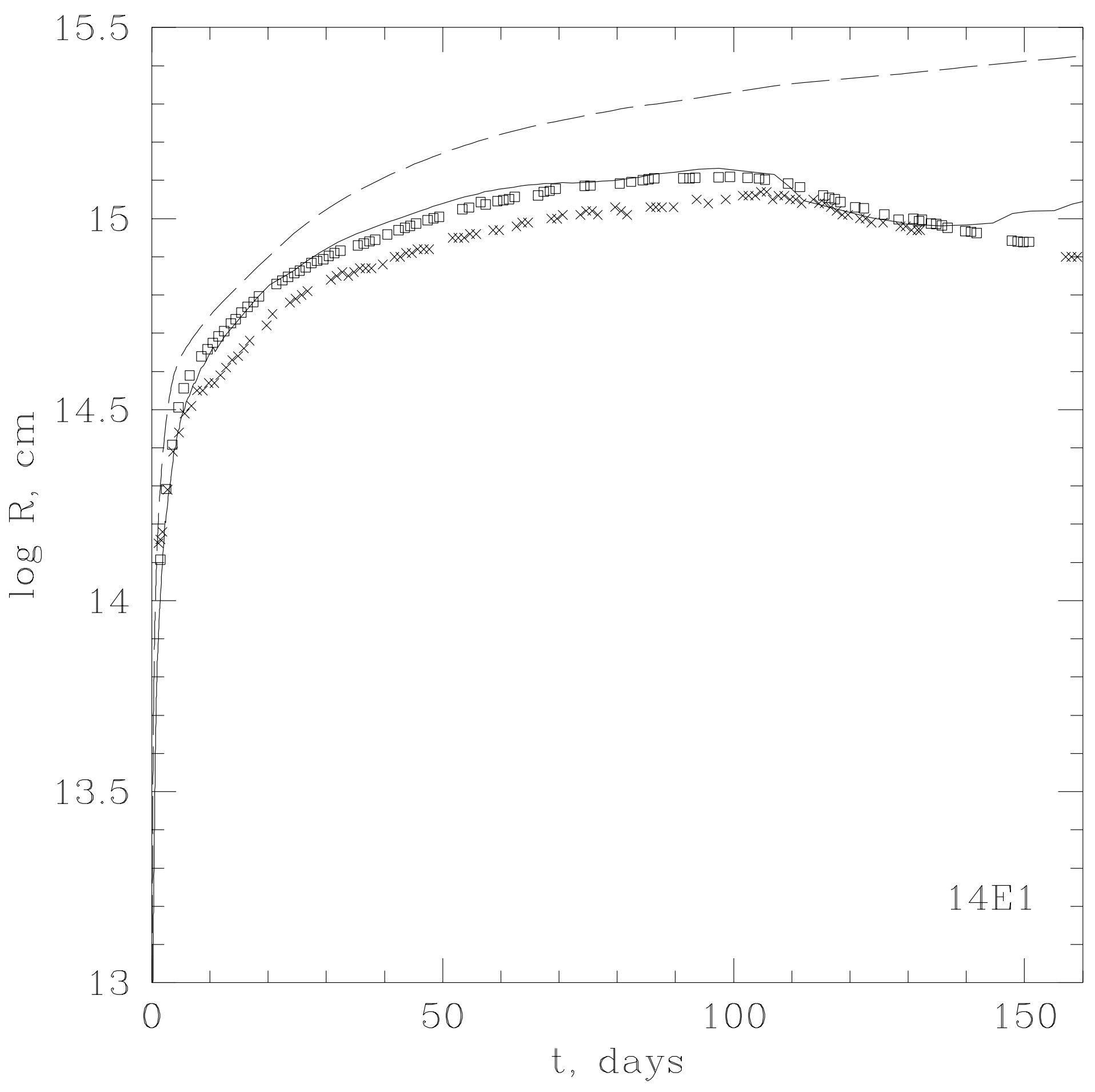




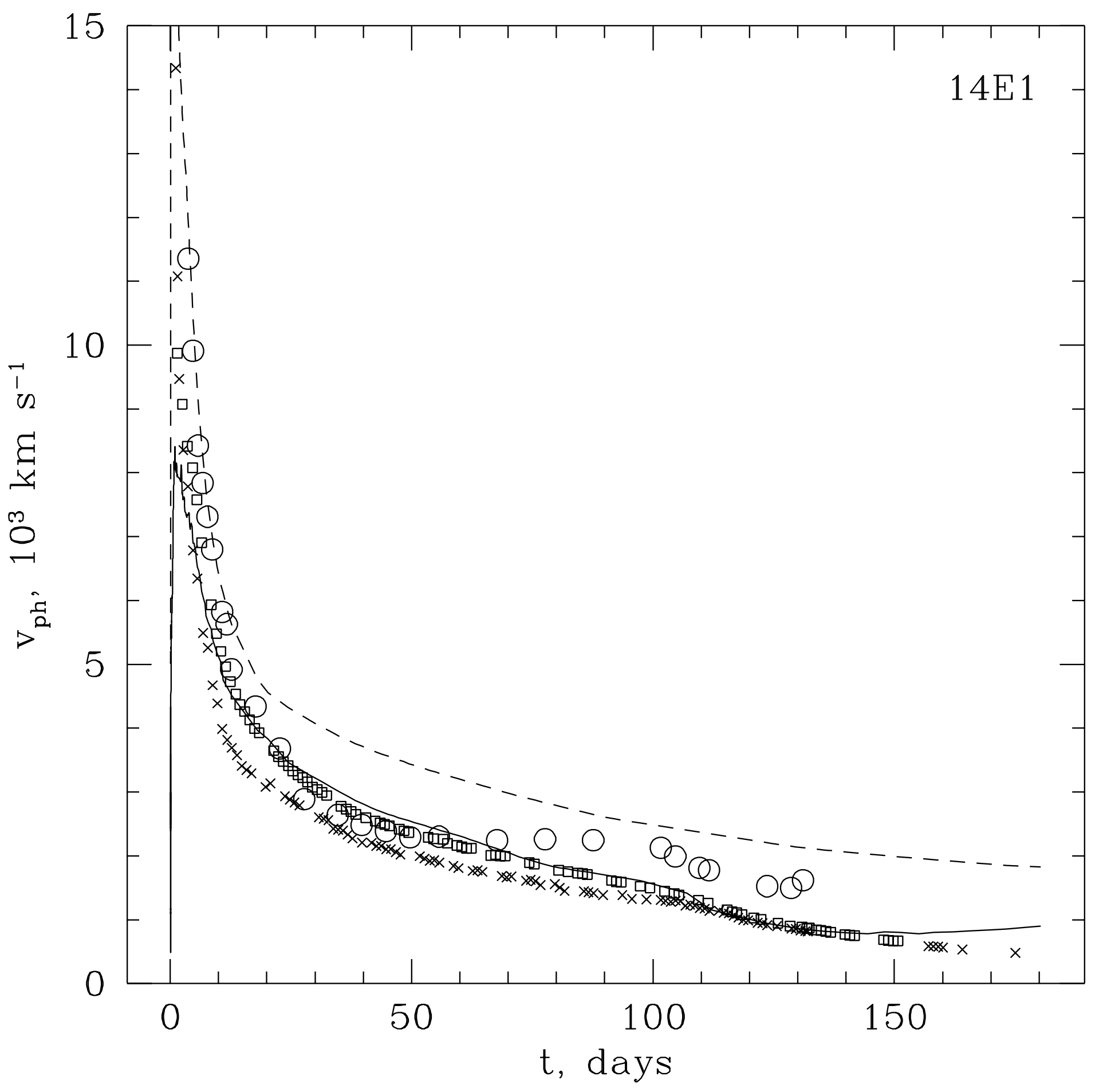




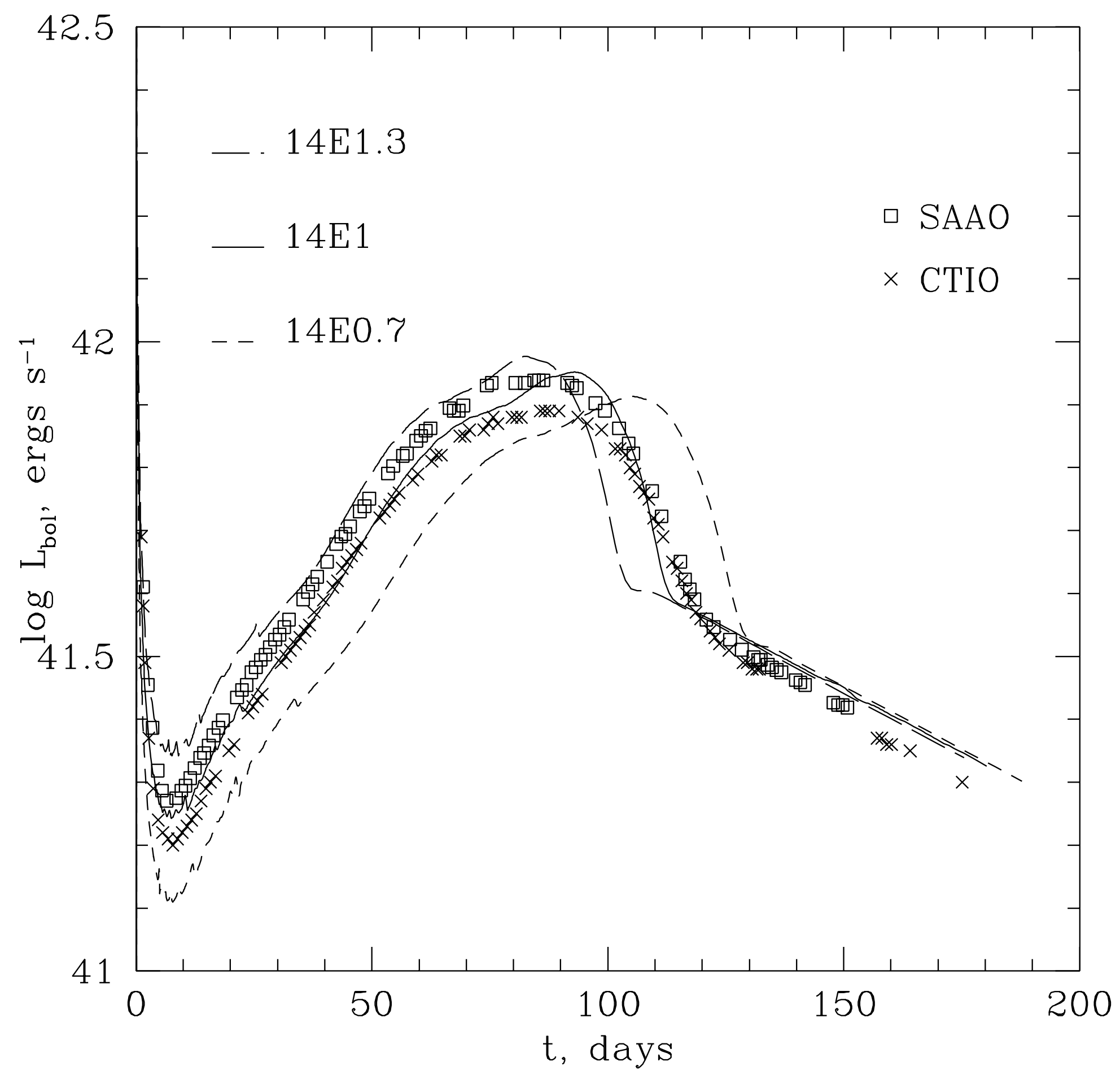




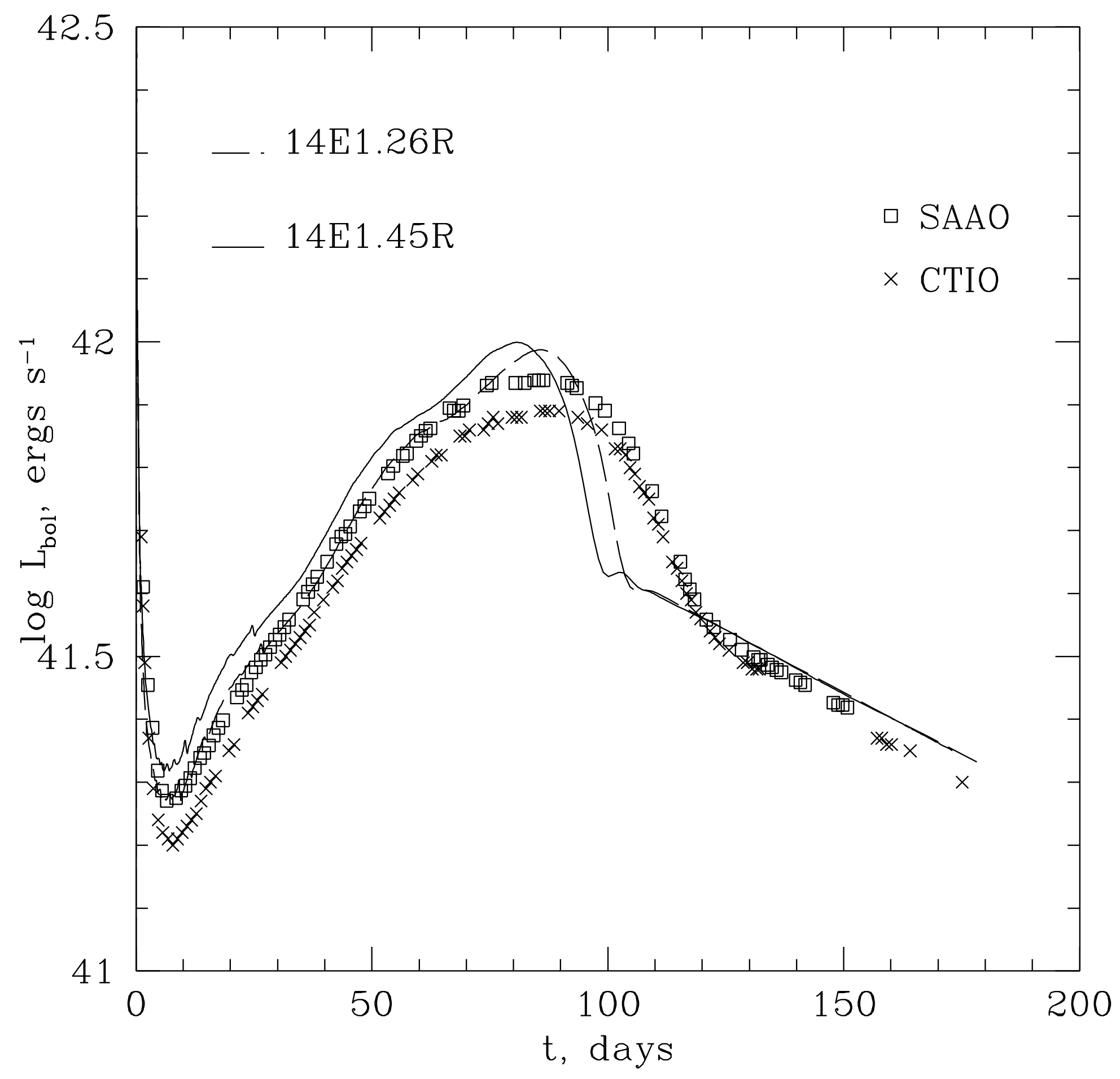




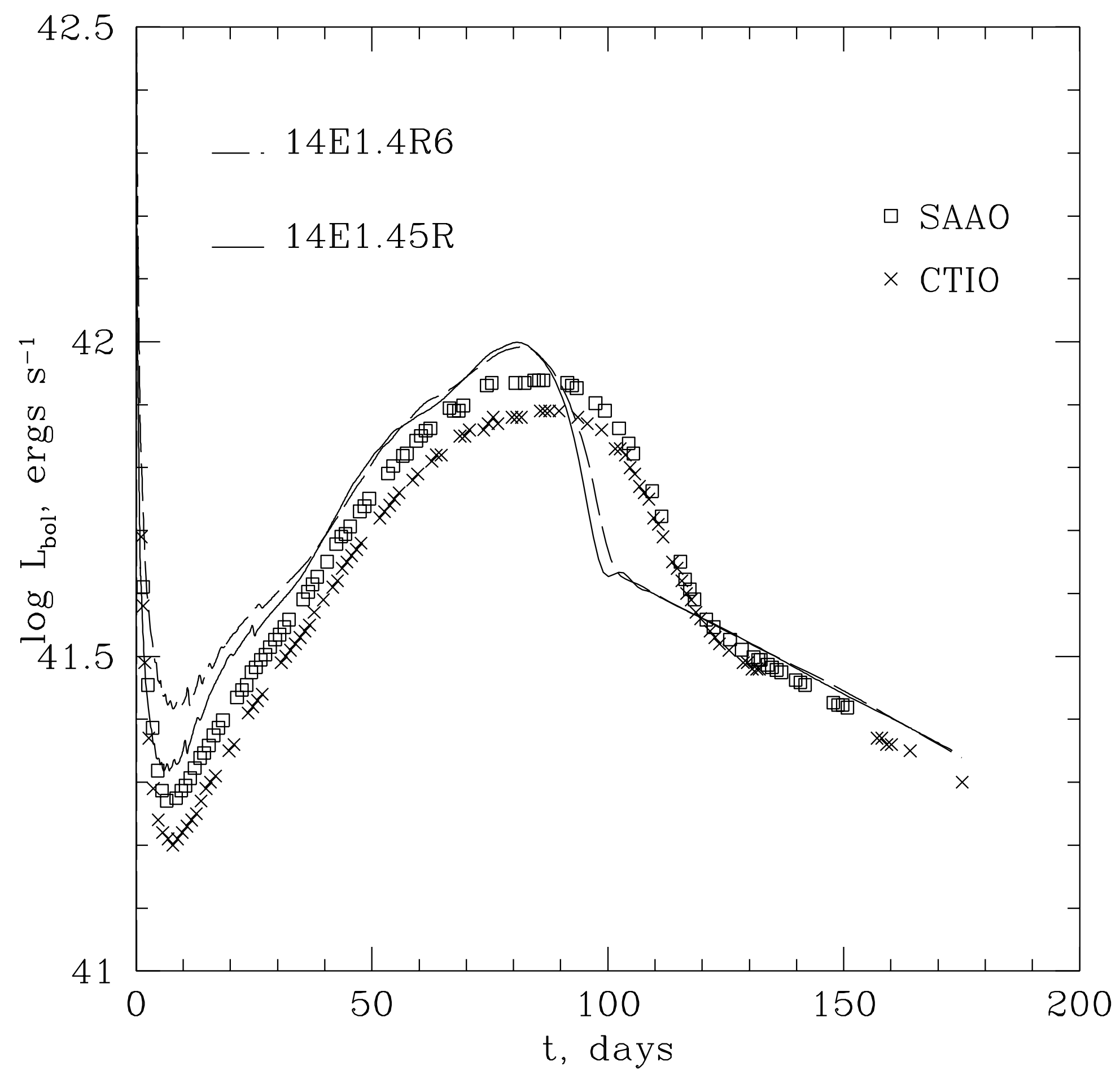




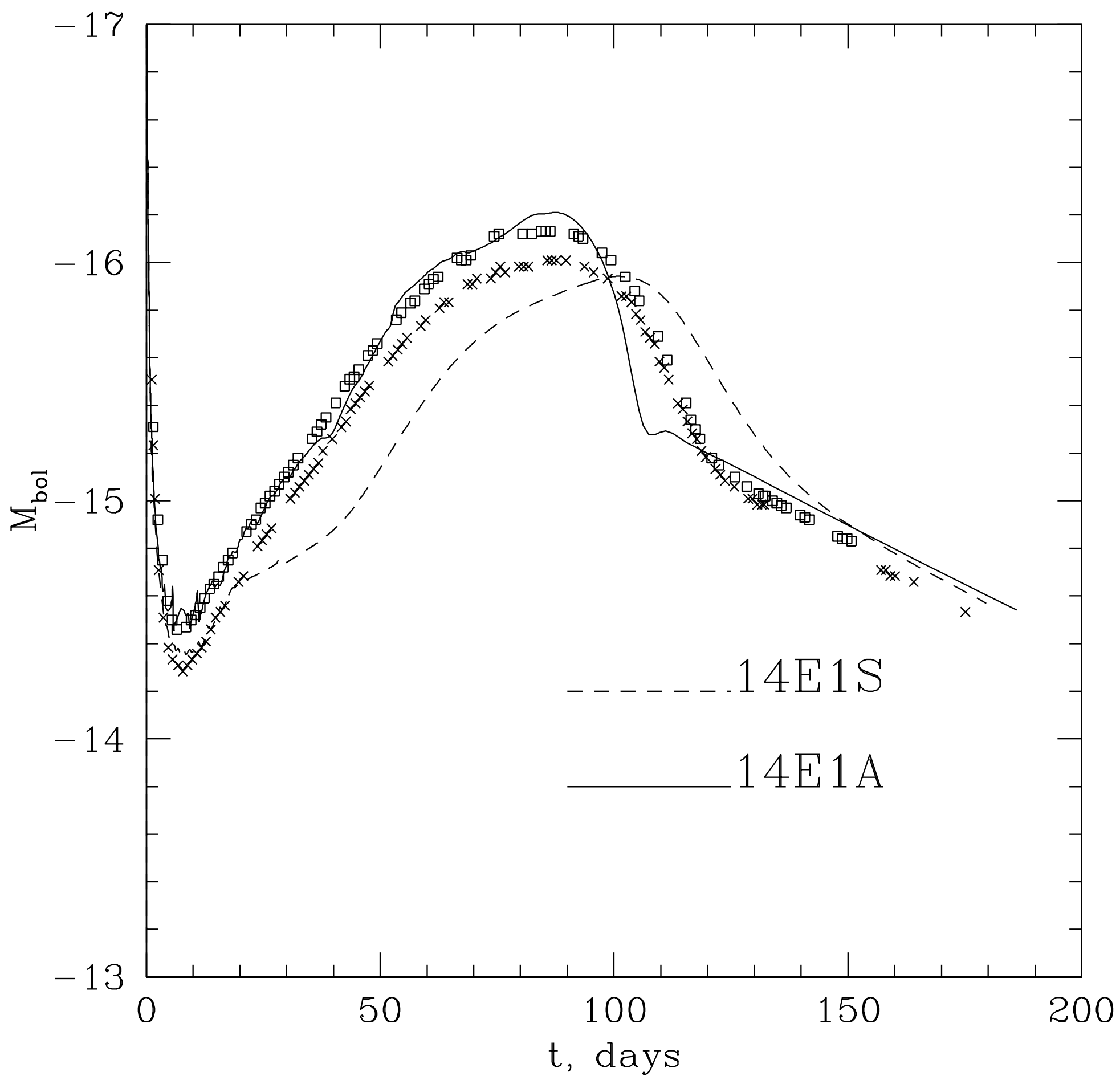




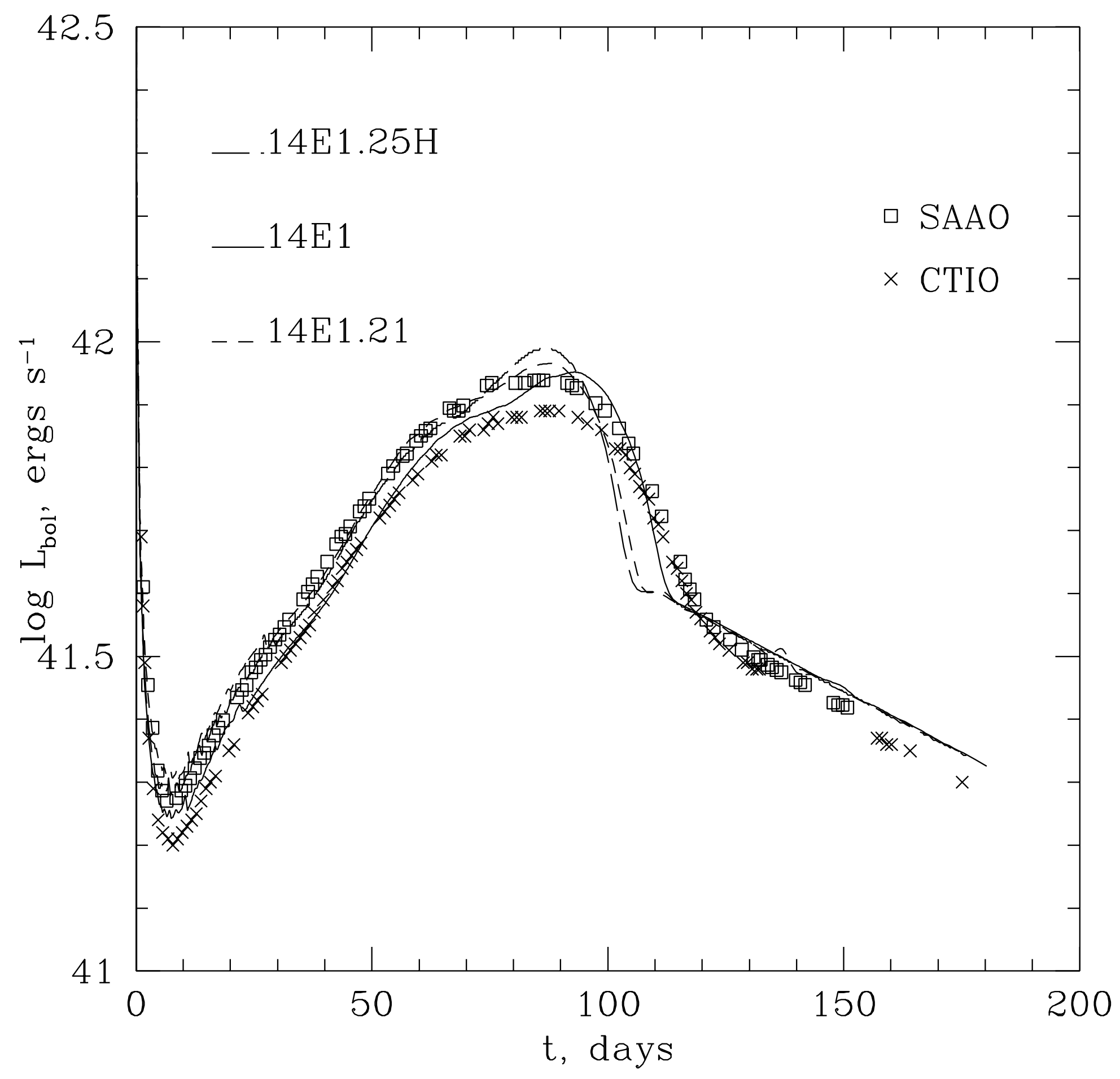

